\title{
ABSOLUTE STABILITY RESULTS FOR WELL-POSED INFINITE-DIMENSIONAL SYSTEMS WITH APPLICATIONS TO LOW-GAIN INTEGRAL CONTROL*
}

\author{
Hartmut Logemann ${ }^{1}$ And Ruth F. Curtain ${ }^{2}$
}

\begin{abstract}
We derive absolute stability results for well-posed infinite-dimensional systems which, in a sense, extend the well-known circle criterion to the case that the underlying linear system is the series interconnection of an exponentially stable well-posed infinite-dimensional system and an integrator and the nonlinearity $\phi$ satisfies a sector condition of the form $\langle\phi(u), \phi(u)-a u\rangle \leq 0$ for some constant $a>0$. These results are used to prove convergence and stability properties of low-gain integral feedback control applied to exponentially stable, linear, well-posed systems subject to actuator nonlinearities. The class of actuator nonlinearities under consideration contains standard nonlinearities which are important in control engineering such as saturation and deadzone.
\end{abstract}

AMS Subject Classification. 93C10, 93C20, 93C25, 93D05, 93D09, 93D10, 93D21.

Received October 18, 1999. Revised March 24, 2000.

\section{INTRODUCTION}

Absolute stability problems and their relations to positive-real conditions have played a prominent role in finite-dimensional systems and control theory and have led to a number of important stability criteria for closedloop systems obtained by applying unity feedback controls to linear dynamical systems subject to static input or output nonlinearities, see, for example, Aizerman and Gantmacher [1], Khalil [13], Lefschetz [14], Leonov et al. [15] and Vidyasagar [28]. Although there is some literature on absolute stability problems in infinite dimensions (for example, Bucci [4], Corduneanu [7], Leonov et al. [15], Logemann [17], Wexler [33, 34]), the number of results available in the literature is fairly limited, in particular for systems with unbounded control and observation.

In this paper we study a certain absolute stability problem for the class of well-posed infinite-dimensional systems which are documented in Salamon [24,25], Staffans [26,27] and Weiss [29-32]. We remark that the class of well-posed, linear, infinite-dimensional systems is rather general: it includes most distributed parameter

Keywords and phrases: Absolute stability, actuator nonlinearities, circle criterion, integral control, positive real, robust tracking, well-posed infinite-dimensional systems.

* This work was supported by the UK-DUTCH Joint Scientific Research Programme and by the UK EPSRC Council (Grant $G R / L 78086)$.

${ }^{1}$ Department of Mathematical Sciences, University of Bath, Claverton Down, Bath BA2 7AY, U.K.; e-mail: hl@maths . bath.ac.uk

2 Mathematics Institute, University of Groningen, P.O. Box 800, 9700 AV Groningen, The Netherlands;

e-mail: R.F.Curtain@math.rug.nl

(c) EDP Sciences, SMAI 2000 


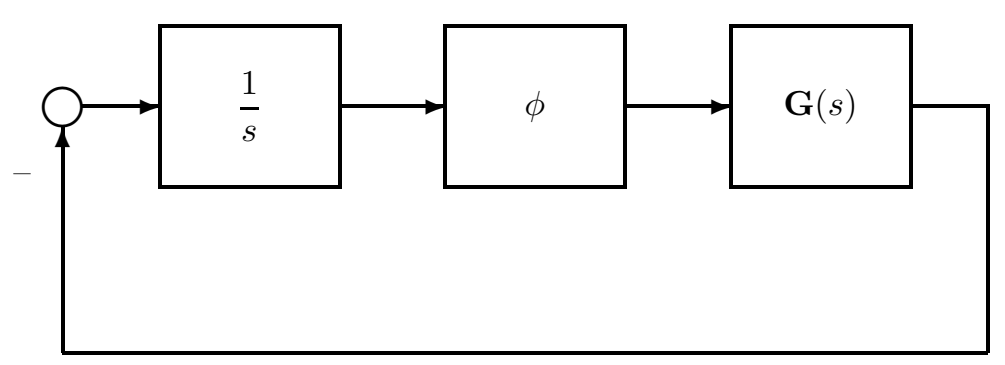

Figure 1

systems and all time-delay systems (retarded and neutral) which are of interest in applications. Consider the system shown in Figure 1, where $\mathbf{G}$ is the transfer function of an exponentially stable, well-posed, infinitedimensional, linear system and $\phi: U \rightarrow U$ is a locally Lipschitz nonlinearity which, for some $a \geq 0$, satisfies the sector condition

$$
\langle\phi(u), \phi(u)-a u\rangle \leq 0, \quad \forall u \in U
$$

where $U$ denotes the input space of the well-posed system which is assumed to be a real Hilbert space. Given $b>0$, we study the absolute stability problem of finding conditions on $\mathbf{G}$ such that the feedback system in Figure 1 is stable for all locally Lipschitz $\phi$ satisfying (1.1) for some $a \in[0, b)$. In Section 3 we show that if $\mathbf{G}(0)$ is invertible and the positive real condition

$$
I+\frac{b}{2}\left(\frac{1}{s} \mathbf{G}(s)+\frac{1}{\bar{s}} \mathbf{G}^{*}(s)\right) \geq 0, \quad s \in \mathbb{C} \text { with } \operatorname{Re} s>0
$$

holds, then, for all locally Lipschitz $\phi$ satisfying (1.1) for some $a \in[0, b)$, the equilibrium of the closed-loop system shown in Figure 1 is stable in the large. Moreover, under suitable extra assumptions on $\phi$, we prove that the equilibrium is semi-globally exponentially stable. These results extend, in a certain sense, a part of the well-known circle criterion, see Remark 3.2, Part (e).

In Section 4 we apply the absolute stability results obtained in Section 3 to the low-gain integral control problem illustrated in Figure 2, where $r \in U$ is the reference vector and $k>0$ is the integral gain.

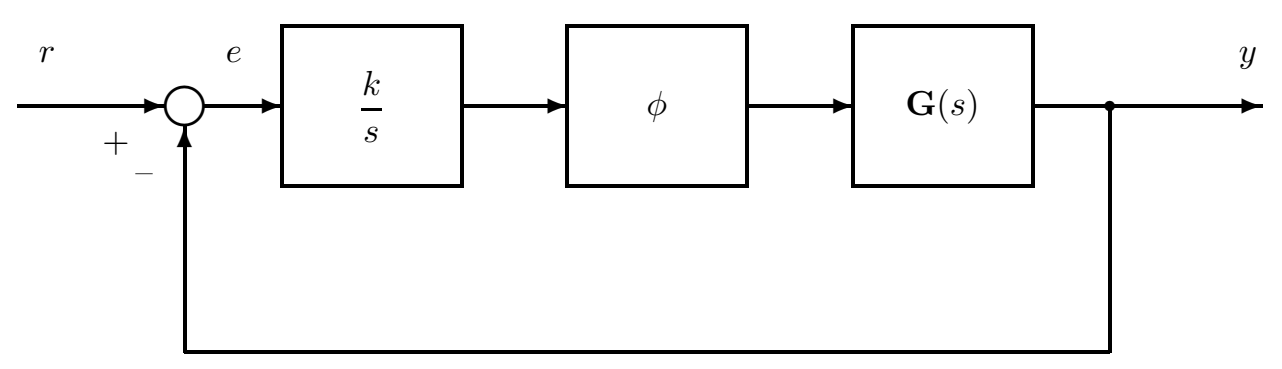

Figure 2

We assume that $U=\mathbb{R}^{m}, \mathbf{G}$ is the transfer function of an exponentially stable well-posed system such that $\mathbf{G}(0)$ is invertible and $\phi: \mathbb{R}^{m} \rightarrow \mathbb{R}^{m}$ is a decoupled nonlinearity of the form

$$
\phi(u)=\left[\phi_{1}\left(u_{1}\right), \phi_{2}\left(u_{2}\right), \ldots, \phi_{m}\left(u_{m}\right)\right]^{T}, \quad \forall u=\left(u_{1}, u_{2}, \ldots, u_{m}\right)^{T} \in \mathbb{R}^{m}
$$


where the functions $\phi_{i}: \mathbb{R} \rightarrow \mathbb{R}$ are non-decreasing and globally Lipschitz with Lipschitz constants $\lambda_{i}>0$. Setting

$$
K:=\sup \{b>0:(1.2) \text { holds }\}, \quad \lambda:=\max \lambda_{i},
$$

we prove that for all $k \in(0, K / \lambda)$ and for all reference vectors $r$ satisfying $[\mathbf{G}(0)]^{-1} r \in \operatorname{im} \phi$, the error $e(\cdot)=$ $r-y(\cdot)$ is in $L^{2}\left(\mathbb{R}_{+}, \mathbb{R}^{m}\right)$. Moreover, we show that if the impulse response of the linear system $(i . e$. the inverse Laplace transform of the transfer function $\mathbf{G}$ ) is a (matrix-valued) Borel measure and if the initial condition of the plant is "sufficiently smooth", then $e(t) \rightarrow 0$ as $t \rightarrow \infty$, i.e., the output $y(t)$ asymptotically tracks the reference vector $r$. Under mild extra assumptions on $r$ and $\phi$, the convergence will be exponentially fast. We remark that these results considerably improve earlier work by Logemann et al. [20] in the sense that (i) our results guarantee better asymptotic and faster convergence properties and (ii) our results are not restricted to single-input single-output regular systems, but apply to the wider class of multivariable well-posed systems.

The paper is organized as follows. Section 2 contains some preliminaries on well-posed infinite-dimensional systems. Section 3 is devoted to a detailed analysis of the absolute stability problem described above. In Section 4 we apply the absolute stability theory developed in Section 3 to derive results on the low-gain integral control problem illustrated in Figure 2. An example of a diffusion process with output delay illustrating our results is given in Section 5. Finally, some technicalities are relegated to the Appendix.

Notation. Let $\mathrm{X}$ be a real or complex Hilbert space; for $\tau \geq 0, \mathbf{R}_{\tau}$ denotes the operator of the right-shift by $\tau$ on $L_{\text {loc }}^{p}\left(\mathbb{R}_{+}, X\right)$, where $\mathbb{R}_{+}:=[0, \infty)$; the truncation operator $\mathbf{P}_{\tau}: L_{\text {loc }}^{p}\left(\mathbb{R}_{+}, X\right) \rightarrow L^{p}\left(\mathbb{R}_{+}, X\right)$ is given by $\left(\mathbf{P}_{\tau} u\right)(t)=u(t)$ if $t \in[0, \tau]$ and $\left(\mathbf{P}_{\tau} u\right)(t)=0$ otherwise; for $\alpha \in \mathbb{R}$, we define the exponentially weighted $L^{p}$-space $L_{\alpha}^{p}\left(\mathbb{R}_{+}, X\right):=\left\{f \in L_{\text {loc }}^{p}\left(\mathbb{R}_{+}, X\right) \mid f(\cdot) \exp (-\alpha \cdot) \in L^{p}\left(\mathbb{R}_{+}, X\right)\right\}$ and endow it with the norm $\|f\|_{p, \alpha}:=$ $\|f(\cdot) \exp (-\alpha \cdot)\|_{L^{p}}$, where $\|\cdot\|_{L^{p}}$ denotes the usual norm in $L^{p}\left(\mathbb{R}_{+}, X\right)$; for $\tau>0, W^{1,2}([0, \tau], X)$ denotes the space of all functions $f:[0, \tau] \rightarrow X$ for which there exists $g \in L^{2}([0, \tau], X)$ such that $f(t)=f(0)+\int_{0}^{t} g(s) d s$ for all $t \in[0, \tau] ; W_{\mathrm{loc}}^{1,2}\left(\mathbb{R}_{+}, X\right)$ denotes the space of all functions $f: \mathbb{R}_{+} \rightarrow X$ such that for all $\tau>0$, the restriction of $f$ to $[0, \tau]$ belongs to $W^{1,2}([0, \tau], X) ; \mathcal{M}$ denotes the space of all $\mathbb{R}^{m \times m}$-valued Borel measures on $\mathbb{R}_{+}$; for $\alpha \in \mathbb{R}$, we define $\mathcal{M}_{\alpha}$ to be the space of all $\mathbb{R}^{m \times m}$-valued Borel measures on $\mathbb{R}_{+}$with the property that the exponentially weighted measure $E \mapsto \int_{E} e^{-\alpha t} \mu(d t)$ belongs to $\mathcal{M}$; for $\alpha \in \mathbb{R}, \mathbb{C}_{\alpha}:=\{s \in \mathbb{C} \mid \operatorname{Re} s>\alpha\}$; $H^{2}\left(\mathbb{C}_{\alpha}, X\right)$ denotes the Hardy-Lebesgue space of square-integrable functions defined on $\mathbb{C}_{\alpha}$ with values in $X$; for a Banach space $Z, H^{\infty}\left(\mathbb{C}_{\alpha}, Z\right)$ denotes the space of bounded holomorphic functions defined on $\mathbb{C}_{\alpha}$ with values in $Z ; \mathcal{B}\left(X_{1}, X_{2}\right)$ denotes the space of bounded linear operators from a Hilbert space $X_{1}$ to a Hilbert space $X_{2}$; we write $\mathcal{B}(X)$ for $\mathcal{B}(X, X)$; the Laplace transform is denoted by $\mathfrak{L}$.

If $X$ is a real Hilbert space, then its complexification is denoted by $X_{c}$. Every vector $z \in X_{c}$ can be uniquely expressed in the form $z=x+i y$, where $x, y \in X$. In particular, $X \subset X_{c}$. The inner product $\langle\cdot, \cdot\rangle$ on $X$ extends in a natural way to a (complex) inner product on $X_{c}$; a similar statement is true for a linear operator $S$ on $X$ (see Halmos [12], p. 150, for details). We shall use the same symbol $\langle\cdot, \cdot\rangle$ (respectively, $S$ ) for the original inner product (respectively, operator) and the associated extensions. A linear operator $S: \operatorname{dom}(S) \subset X_{c} \rightarrow Y_{c}$, where $Y$ is a real Hilbert space, is called real, if $S x \in Y$ for all $x \in \operatorname{dom}(S) \cap X$.

\section{PRELiminaries ON WELl-POSED SYSTEMS}

We assemble some fundamental facts pertaining to well-posed linear systems and regular linear systems and tailored to later requirements: the reader is referred to Salamon [24,25], Staffans [26,27] and Weiss [29-32] for full details.

Well-posed systems. The concept of a well-posed linear system which will be used in this paper was introduced in [32]; an equivalent definition can be found in [24]. Let $U, X$ and $Y$ be real Hilbert spaces and let $\boldsymbol{\Sigma}=(\mathbf{T}, \mathbf{\Phi}, \mathbf{\Psi}, \mathbf{F})$ be a well-posed linear system with state space $X$, input space $U$ and output space $Y$, i.e.

$\mathbf{T}=\left(\mathbf{T}_{t}\right)_{t \geq 0}$ is a $C_{0}$-semigroup of bounded linear operators on $X$; 
$\mathbf{\Phi}=\left(\boldsymbol{\Phi}_{t}\right)_{t \geq 0}$ is a family of bounded linear operators from $L^{2}\left(\mathbb{R}_{+}, U\right)$ to $X$ such that, for all $\tau, t \geq 0$,

$$
\boldsymbol{\Phi}_{\tau+t}\left(\mathbf{P}_{\tau} u+\mathbf{R}_{\tau} v\right)=\mathbf{T}_{t} \boldsymbol{\Phi}_{\tau} u+\boldsymbol{\Phi}_{t} v, \quad \forall u, v \in L^{2}\left(\mathbb{R}_{+}, U\right) ;
$$

$\boldsymbol{\Psi}=\left(\boldsymbol{\Psi}_{t}\right)_{t \geq 0}$ is a family of bounded linear operators from $X$ to $L^{2}\left(\mathbb{R}_{+}, Y\right)$ such that $\mathbf{\Psi}_{0}=0$ and, for all $\tau, t \geq 0$,

$$
\mathbf{\Psi}_{\tau+t} x^{0}=\mathbf{P}_{\tau} \boldsymbol{\Psi}_{\tau} x^{0}+\mathbf{R}_{\tau} \boldsymbol{\Psi}_{t} \mathbf{T}_{\tau} x^{0}, \quad \forall x^{0} \in X ;
$$

$\mathbf{F}=\left(\mathbf{F}_{t}\right)_{t \geq 0}$ is a family of bounded linear operators from $L^{2}\left(\mathbb{R}_{+}, U\right)$ to $L^{2}\left(\mathbb{R}_{+}, Y\right)$ such that $\mathbf{F}_{0}=0$ and, for all $\tau, t \geq 0$,

$$
\mathbf{F}_{\tau+t}\left(\mathbf{P}_{\tau} u+\mathbf{R}_{\tau} v\right)=\mathbf{P}_{\tau} \mathbf{F}_{\tau} u+\mathbf{R}_{\tau}\left(\mathbf{\Psi}_{t} \boldsymbol{\Phi}_{\tau} u+\mathbf{F}_{t} v\right), \quad \forall u, v \in L^{2}\left(\mathbb{R}_{+}, U\right) .
$$

For an input $u \in L_{\text {loc }}^{2}\left(\mathbb{R}_{+}, U\right)$ and initial state $x^{0} \in X$, the associated state function $x \in C\left(\mathbb{R}_{+}, X\right)$ and output function $y \in L_{\text {loc }}^{2}\left(\mathbb{R}_{+}, Y\right)$ of $\boldsymbol{\Sigma}$ are given by

$$
\begin{aligned}
& x(t)=\mathbf{T}_{t} x^{0}+\mathbf{\Phi}_{t} \mathbf{P}_{t} u \\
& \mathbf{P}_{t} y=\mathbf{\Psi}_{t} x^{0}+\mathbf{F}_{t} \mathbf{P}_{t} u .
\end{aligned}
$$

$\boldsymbol{\Sigma}$ is said to be exponentially stable if the semigroup $\mathbf{T}$ is exponentially stable:

$$
\omega(\mathbf{T}):=\lim _{t \rightarrow \infty} \frac{1}{t} \ln \left\|\mathbf{T}_{t}\right\|<0 .
$$

$\Psi_{\infty}$ and $\mathbf{F}_{\infty}$ will denote the unique operators $X \rightarrow L_{\mathrm{loc}}^{2}\left(\mathbb{R}_{+}, Y\right)$ and $L_{\mathrm{loc}}^{2}\left(\mathbb{R}_{+}, U\right) \rightarrow L_{\mathrm{loc}}^{2}\left(\mathbb{R}_{+}, Y\right)$, respectively, satisfying

$$
\mathbf{\Psi}_{\tau}=\mathbf{P}_{\tau} \mathbf{\Psi}_{\infty}, \quad \mathbf{F}_{\tau}=\mathbf{P}_{\tau} \mathbf{F}_{\infty} ; \quad \forall \tau \geq 0 .
$$

For any $\alpha>\omega(\mathbf{T}), \mathbf{\Psi}_{\infty}$ is a bounded operator from $X$ into $L_{\alpha}^{2}\left(\mathbb{R}_{+}, Y\right)$ and $\mathbf{F}_{\infty}$ maps $L_{\alpha}^{2}\left(\mathbb{R}_{+}, U\right)$ boundedly into $L_{\alpha}^{2}\left(\mathbb{R}_{+}, Y\right)$. If $\boldsymbol{\Sigma}$ is exponentially stable, then the operators $\boldsymbol{\Phi}_{t}, \boldsymbol{\Psi}_{t}$ and $\mathbf{F}_{t}$ are uniformly bounded. Since $\mathbf{P}_{\tau} \mathbf{F}_{\infty}=\mathbf{P}_{\tau} \mathbf{F}_{\infty} \mathbf{P}_{\tau}$ for all $\tau \geq 0, \mathbf{F}_{\infty}$ is a causal operator, called the input-output operator of $\boldsymbol{\Sigma}$.

Transfer functions. Weiss [29] has established that if $\alpha>\omega(\mathbf{T})$, then there exists a unique holomorphic function $\mathbf{G}: \mathbb{C}_{\omega(\mathbf{T})} \rightarrow \mathcal{B}\left(U_{c}, Y_{c}\right)$ such that

$$
\mathbf{G}(s)(\mathfrak{L} u)(s)=\left[\mathfrak{L}\left(\mathbf{F}_{\infty} u\right)\right](s), \quad \forall s \in \mathbb{C}_{\alpha}, \quad \forall u \in L_{\alpha}^{2}\left(\mathbb{R}_{+}, U\right),
$$

where $\mathfrak{L}$ denotes Laplace transform. In particular, $\mathbf{G}$ is bounded on $\mathbb{C}_{\alpha}$ for all $\alpha>\omega(\mathbf{T})$. Moreover, for all $s \in(\omega(\mathbf{T}), \infty), \mathbf{G}(s)$ is a real operator. The function $\mathbf{G}$ is called the transfer function of $\boldsymbol{\Sigma}$.

$\boldsymbol{\Sigma}$ and its transfer function $\mathbf{G}$ are said to be regular if there exists a linear operator $D$ such that

$$
\lim _{s \rightarrow \infty, s \in \mathbb{R}} \mathbf{G}(s) u=D u, \quad \forall u \in U,
$$

in which case, by the principle of uniform boundedness, it follows that $D \in \mathcal{B}(U, Y)$ (in particular, $D$ is real). The operator $D$ is called the feedthrough operator of $\boldsymbol{\Sigma}$.

Control and observation operators. The generator of $\mathbf{T}$ is denoted by $A$ with domain $\operatorname{dom}(A)$. Let $X_{1}$ be the space $\operatorname{dom}(A)$ endowed with the graph norm. The norm on $X$ is denoted by $\|\cdot\|$, whilst $\|\cdot\|_{1}$ denotes the graph norm. Let $X_{-1}$ be the completion of $X$ with respect to the norm $\|x\|_{-1}=\left\|(\lambda I-A)^{-1} x\right\|$, where $\lambda \in \varrho(A)$ is any fixed element of the resolvent set $\varrho(A)$ of $A$. Then $X_{1} \subset X \subset X_{-1}$ and the canonical injections are bounded and dense. The semigroup $\mathbf{T}$ can be restricted to a $C_{0}$-semigroup on $X_{1}$ and extended to a $C_{0}$-semigroup on $X_{-1}$. The exponential growth constant is the same on all three spaces. The generator on $X_{-1}$ is an extension of $A$ to $X$ (which is bounded as an operator from $X$ to $X_{-1}$ ). We shall use the same 
symbol $\mathbf{T}$ (respectively, $A$ ) for the original semigroup (respectively, its generator) and the associated restrictions and extensions. With this convention, we may write $A \in \mathcal{B}\left(X, X_{-1}\right)$. Considered as a generator on $X_{-1}$, the domain of $A$ is $X$.

By a representation theorem due to Salamon [24] (see also Weiss [30,31]), there exist unique operators $B \in \mathcal{B}\left(U, X_{-1}\right)$ and $C \in \mathcal{B}\left(X_{1}, Y\right)$ (the control operator and the observation operator of $\boldsymbol{\Sigma}$, respectively) such that, for all $t \geq 0, u \in L_{\mathrm{loc}}^{2}\left(\mathbb{R}_{+}, U\right)$ and $x^{0} \in X_{1}$,

$$
\mathbf{\Phi}_{t} \mathbf{P}_{t} u=\int_{0}^{t} \mathbf{T}_{t-\tau} B u(\tau) d \tau \quad \text { and } \quad\left(\Psi_{\infty} x^{0}\right)(t)=C \mathbf{T}_{t} x^{0} .
$$

The so-called Lebesgue extension of $C$, denoted by $C_{L}$, is defined by

$$
C_{L} x^{0}=\lim _{t \rightarrow 0} C \frac{1}{t} \int_{0}^{t} \mathbf{T}_{\tau} x^{0} d \tau
$$

where $\operatorname{dom}\left(C_{L}\right)$ is the set of all those $x^{0} \in X$ for which the above limit exists (see [31]). Clearly $X_{1} \subset \operatorname{dom}\left(C_{L}\right)$ $\subset X$. Furthermore, for any $x^{0} \in X$, we have that $\mathbf{T}_{t} x^{0} \in \operatorname{dom}\left(C_{L}\right)$ for almost every $t \geq 0$ and

$$
\left(\mathbf{\Psi}_{\infty} x^{0}\right)(t)=C_{L} \mathbf{T}_{t} x^{0}, \quad \text { a.e. } t \geq 0 .
$$

$B$ is said to be bounded if it is so as a map from the input space $U$ to the state space $X$, otherwise, $B$ is said to be unbounded. $C$ is said to be bounded if it can be extended continuously to $X$, otherwise, $C$ is said to be unbounded. If $\mathbf{T}$ is exponentially stable, then there exist constants $\beta_{1}, \beta_{2}>0$ such that, for all $t \geq 0$, $u \in L^{2}\left(\mathbb{R}_{+}, U\right)$ and $x^{0} \in X$,

$$
\begin{aligned}
& \left\|\mathbf{\Phi}_{t} \mathbf{P}_{t} u\right\|=\left\|\int_{0}^{t} \mathbf{T}_{t-\tau} B u(\tau) d \tau\right\| \leq \beta_{1}\|u\|_{L^{2}(0, t ; U)}, \\
& \left\|\mathbf{\Psi}_{\infty} x^{0}\right\|_{L^{2}(0, t ; Y)}=\left(\int_{0}^{t}\left\|C \mathbf{T}_{\tau} x^{0}\right\|^{2} d \tau\right)^{1 / 2} \leq \beta_{2}\left\|x^{0}\right\| .
\end{aligned}
$$

For any $x^{0} \in X$ and $u \in L_{\text {loc }}^{2}\left(\mathbb{R}_{+}, U\right)$, the state trajectory $x(\cdot)$ defined by (2.1a) satisfies the equation

$$
\dot{x}(t)=A x(t)+B u(t), \quad x(0)=x^{0}, \quad \text { a.e. } t \geq 0 .
$$

The derivative on the left-hand side of (2.5) has, of course, to be understood in $X_{-1}$. In other words, if we consider the initial-value problem (2.5) in the space $X_{-1}$, then for any $x^{0} \in X$ and $u \in L_{\text {loc }}^{2}\left(\mathbb{R}_{+}, U\right),(2.5)$ has a unique strong solution (in the sense of Pazy [22], p. 109) given by the variation of parameters formula

$$
t \mapsto x(t)=\mathbf{T}_{t} x^{0}+\int_{0}^{t} \mathbf{T}_{t-\tau} B u(\tau) d \tau .
$$

It has been shown in [24] that for any $x^{0} \in X_{1}, u \in W_{\text {loc }}^{1,2}\left(\mathbb{R}_{+}, U\right)$ with $u(0)=0$ and $s_{0} \in \varrho(A)$, the output function $y(\cdot)$ defined by $(2.1 \mathrm{~b})$ can be expressed as

$$
y(t)=C\left[\mathbf{T}_{t} x^{0}+\int_{0}^{t} \mathbf{T}_{t-\tau} B u(\tau) d \tau-\left(s_{0} I-A\right)^{-1} B u(t)\right]+\mathbf{G}\left(s_{0}\right) u(t), \text { a.e. } t \geq 0 .
$$

If $\boldsymbol{\Sigma}$ is regular (with feedthrough $D$ ), then the state trajectory $x(\cdot)$ defined by $\left(2.1\right.$ a) satisfies $x(t) \in \operatorname{dom}\left(C_{L}\right)$ for almost every $t \geq 0$ and the output $y(t)$ given by $(2.1 \mathrm{~b})$ can be written in the familiar form

$$
y(t)=C_{L} x(t)+D u(t), \quad \text { a.e. } t \geq 0 .
$$


We know from [10] and [24] that for all $s, s_{0} \in \varrho(A), s \neq s_{0}$

$$
\frac{1}{s-s_{0}}\left(\mathbf{G}(s)-\mathbf{G}\left(s_{0}\right)\right)=-C(s I-A)^{-1}\left(s_{0} I-A\right)^{-1} B .
$$

Moreover, it has been demonstrated in [29] that, if $\boldsymbol{\Sigma}$ is regular (with feedthrough $D$ ), then $(s I-A)^{-1} B U \subset$ $\operatorname{dom}\left(C_{L}\right)$ for all $s \in \varrho(A)$ and the transfer function $\mathbf{G}$ can be expressed as

$$
\mathbf{G}(s)=C_{L}(s I-A)^{-1} B+D, \quad \forall s \in \mathbb{C}_{\omega(\mathbf{T})},
$$

which is familiar from finite-dimensional systems theory. The operators $A, B, C$ (and $D$, in the regular case) are called the generating operators of $\boldsymbol{\Sigma}$. Note that in the non-regular case, the generating operators do not completely determine the system $\boldsymbol{\Sigma}$, since $A, B$ and $C$ determine the input-output operator $\mathbf{F}_{\infty}$ only up to an additive constant (see (2.9)).

Four technical lemmas. In the following let $\boldsymbol{\Sigma}=(\mathbf{T}, \boldsymbol{\Phi}, \mathbf{\Psi}, \mathbf{F})$ be a well-posed linear system with state space $X$, input space $U$, output space $Y$, generating operators $A, B$ and $C$, input-output operator $\mathbf{F}_{\infty}$ and transfer function $\mathbf{G}$. We state four lemmas on the asymptotic behaviour and the regularity of the solutions to (2.1) and on the existence and uniqueness of solutions for a certain nonlinear feedback system with (2.1) in the forward loop.

Lemma 2.1. Let $x^{0} \in X$. If $u \in L_{\text {loc }}^{2}\left(\mathbb{R}_{+}, U\right)$ and $u^{\infty} \in U$ are such that $u-u^{\infty} \in L_{\alpha}^{2}\left(\mathbb{R}_{+}, U\right)$ for some $\alpha>\omega(\mathbf{T})$, then the output $y$ of $\boldsymbol{\Sigma}$ (given by (2.1b)) satisfies

$$
y-\mathbf{G}(0) u^{\infty} \in L_{\alpha}^{2}\left(\mathbb{R}_{+}, Y\right) .
$$

Proof: Since $\alpha>\omega(\mathbf{T})$, the function $t \mapsto C_{L} \mathbf{T}_{t} x^{0}$ is in $L_{\alpha}^{2}\left(\mathbb{R}_{+}, Y\right)$. Combining this with the identity

$$
y(t)=C_{L} \mathbf{T}_{t} x^{0}+\left(\mathbf{F}_{\infty} u\right)(t), \quad \text { a.e. } t \geq 0,
$$

it follows that it is sufficient to show that

$$
\mathbf{F}_{\infty} u-y^{\infty} \in L_{\alpha}^{2}\left(\mathbb{R}_{+}, Y\right)
$$

where $y^{\infty}:=\mathbf{G}(0) u^{\infty}$. Trivially, we have

$$
\mathbf{F}_{\infty} u-y^{\infty}=\mathbf{F}_{\infty}\left(u-u^{\infty}\right)+\mathbf{F}_{\infty}\left(u^{\infty}\right)-y^{\infty} .
$$

Since $u-u^{\infty} \in L_{\alpha}^{2}\left(\mathbb{R}_{+}, U\right)$ for some $\alpha>\omega(\mathbf{T})$, we may conclude that $\mathbf{F}_{\infty}\left(u-u^{\infty}\right) \in L_{\alpha}^{2}\left(\mathbb{R}_{+}, Y\right)$. Therefore, by (2.11), the claim follows if we can show that

$$
\mathbf{F}_{\infty}\left(u^{\infty}\right)-y^{\infty} \in L_{\alpha}^{2}\left(\mathbb{R}_{+}, Y\right) .
$$

To this end take the Laplace transform of $\mathbf{F}_{\infty}\left(u^{\infty}\right)-y^{\infty}$ to obtain

$$
\left(\mathfrak{L}\left(\mathbf{F}_{\infty}\left(u^{\infty}\right)-y^{\infty}\right)\right)(s)=\frac{1}{s} \mathbf{G}(s) u^{\infty}-\frac{1}{s} y^{\infty}=\frac{1}{s}(\mathbf{G}(s)-\mathbf{G}(0)) u^{\infty} .
$$

It is clear that the function $s \mapsto(\mathbf{G}(s)-\mathbf{G}(0)) u^{\infty} / s$ is in $H^{2}\left(\mathbb{C}_{\alpha}, Y\right)$ and therefore, appealing to a well-known theorem due to Paley and Wiener, it follows from (2.13) that $\mathbf{F}_{\infty}\left(u^{\infty}\right)-y^{\infty} \in L_{\alpha}^{2}\left(\mathbb{R}_{+}, Y\right)$, which is (2.12).

Lemma 2.2. Suppose that $\mathbf{T}$ is exponentially stable. Then, for all $x^{0} \in X$ and $u \in L^{2}\left(\mathbb{R}_{+}, U\right)$, the solution $x(\cdot)$ of the initial-value problem (2.5) satisfies

$$
\lim _{t \rightarrow \infty}\|x(t)\|=0, \quad x \in L^{2}\left(\mathbb{R}_{+}, X\right) .
$$


Proof: Let $x_{0} \in X$ and $u \in L^{2}\left(\mathbb{R}_{+}, U\right)$ and assume that $\mathbf{T}$ is exponentially stable. It has been shown in [21], Lemma 2.2, that under these assumptions $x \in L^{2}\left(\mathbb{R}_{+}, X\right)$. It remains to show that $\lim _{t \rightarrow \infty}\|x(t)\|=0$. To this end note that by the exponential stability of $\mathbf{T}$ and (2.3), there exists $\beta>0$ such that

$$
\left\|\int_{s}^{t} \mathbf{T}_{t-\tau} B u(\tau) d \tau\right\| \leq \beta\left(\int_{s}^{t}\|u(\tau)\|^{2} d \tau\right)^{1 / 2}, \quad t \geq s \geq 0 .
$$

Since $u \in L^{2}\left(\mathbb{R}_{+}, U\right)$, there exists $s_{1} \geq 0$ such that

$$
\int_{s}^{t}\|u(\tau)\|^{2} d \tau \leq \varepsilon^{2} / 4 \beta^{2}, \quad t \geq s \geq s_{1} .
$$

Let $\varepsilon>0$. By the exponential stability of $\mathbf{T}$ there exists $s_{2} \geq 0$ such that

$$
\left\|\mathbf{T}_{t} x\left(s_{1}\right)\right\| \leq \varepsilon / 2, \quad \forall t \geq s_{2} .
$$

Now

and hence, by combining (2.14-2.16), we obtain for all $t \geq s_{1}+s_{2}$

$$
x(t)=\mathbf{T}_{t-s_{1}} x\left(s_{1}\right)+\int_{s_{1}}^{t} \mathbf{T}_{t-\tau} B u(\tau) d \tau,
$$

$$
\|x(t)\| \leq\left\|\mathbf{T}_{t-s_{1}} x\left(s_{1}\right)\right\|+\beta\left(\int_{s_{1}}^{t}\|u(\tau)\|^{2} d \tau\right)^{1 / 2} \leq \varepsilon / 2+\varepsilon / 2=\varepsilon,
$$

showing that $\lim _{t \rightarrow \infty}\|x(t)\|=0$.

The following lemma can be found in Salamon [25] (more precisely, it is part of Lem. 2.5 in [25]).

Lemma 2.3. If $x^{0} \in X$ and $u \in W_{\mathrm{loc}}^{1,2}\left(\mathbb{R}_{+}, U\right)$ are such that $A x^{0}+B u(0) \in X$, then the solution $x(\cdot)$ of the initial-value problem (2.5) is continuously differentiable in $X$.

Suppose that $Y=U$ and consider the following nonlinear system

$$
\begin{aligned}
& \dot{x}(t)=A x(t)+B \phi(u(t)), \quad x(0)=x^{0} \in X \\
& \dot{u}(t)=k\left\{r-C_{L} \mathbf{T}_{t} x^{0}-\left[\mathbf{F}_{\infty}(\phi(u))\right](t)\right\}, \quad u(0)=u^{0} \in U,
\end{aligned}
$$

where the reference vector $r \in U$, the gain parameter $k \in \mathbb{R}$ and the nonlinearity $\phi: U \rightarrow U$ satisfies a local Lipschitz condition, that is, for every bounded set $W \subset U$ there exists a constant $l \geq 0$ such that

$$
\|\phi(u)-\phi(v)\| \leq l\|u-v\|, \quad \forall u, v \in W .
$$

Of course, $y(t):=C_{L} \mathbf{T}_{t} x^{0}+\left[\mathbf{F}_{\infty}(\phi(u))\right](t)$ is the output of the well-posed system $\boldsymbol{\Sigma}$ corresponding to the initial condition $x(0)=x^{0}$ and the input $\phi \circ u$, and so (2.17b) may be written in the compact form $\dot{u}(t)=k(r-y(t))$.

For $T \in(0, \infty]$, a continuous function

$$
[0, T) \rightarrow X \times U, \quad t \mapsto(x(t), u(t))
$$

is a solution of $(2.17)$ if $(x(\cdot), u(\cdot))$ is absolutely continuous as a $\left(X_{-1} \times U\right)$-valued function, $(x(0), u(0))=\left(x^{0}, u^{0}\right)$ and the differential equations in (2.17) are satisfied almost everywhere on $[0, a)$, where the derivative in (2.17a) should be interpreted in the space $X_{-1}{ }^{3}$. An application of a well-known result on abstract Cauchy problems

\footnotetext{
${ }^{3}$ Being a Hilbert space $X_{-1} \times U$ is reflexive, and hence any absolutely continuous $\left(X_{-1} \times U\right)$-valued function is a.e. differentiable and can be recovered from its derivative by integration, see [3], Theorem 3.1 (p. 10).
} 
(see Pazy [22], Th. 2.4, p. 107) shows that a continuous $(X \times U)$-valued function $(x(\cdot), u(\cdot))$ is a solution of $(2.17)$ if, and only if, it satisfies the following integrated version of $(2.17)$

$$
\begin{aligned}
& x(t)=\mathbf{T}_{t} x^{0}+\int_{0}^{t} \mathbf{T}_{t-\tau} B \phi(u(\tau)) d \tau, \\
& u(t)=u^{0}+k \int_{0}^{t}\left[r-C_{L} \mathbf{T}_{\tau} x^{0}-\left(\mathbf{F}_{\infty} \phi(u)\right)(\tau)\right] d \tau .
\end{aligned}
$$

The next result asserts that (2.17) has a unique solution: under the assumption that $\boldsymbol{\Sigma}$ is regular, this has been established in [20] (see Prop. 3.1 in [20]). An inspection of the proof in [20] shows that it carries over to well-posed systems without any changes.

Lemma 2.4. Let $\phi: U \rightarrow U$ be locally Lipschitz. For each $\left(x^{0}, u^{0}\right) \in X \times U$, there exists a unique solution $(x(\cdot), u(\cdot))$ of (2.17) defined on a maximal interval $[0, T)$. If $T<\infty$, then

$$
\limsup _{t \rightarrow T}(\|x(t)\|+\|u(t)\|)=\infty
$$

If $\phi$ is globally Lipschitz, then $T=\infty$.

\section{Absolute stability Results}

In the following let $\boldsymbol{\Sigma}=(\mathbf{T}, \boldsymbol{\Phi}, \mathbf{\Psi}, \mathbf{F})$ be a well-posed linear system with state space $X$, input space $U$, output space $Y=U$, generating operators $A, B$ and $C$, input-output operator $\mathbf{F}_{\infty}$ and transfer function $\mathbf{G}$. In this section we consider the nonlinear system (2.17) with $r=0$ and $k=1$, i.e.,

$$
\begin{aligned}
& \dot{x}(t)=A x(t)+B \phi(u(t)), \quad x(0)=x^{0} \in X \\
& \dot{u}(t)=-C_{L} \mathbf{T}_{t} x^{0}-\left[\mathbf{F}_{\infty}(\phi(u))\right](t), \quad u(0)=u^{0} \in U .
\end{aligned}
$$

We assume that $\phi$ is locally Lipschitz (in the sense of Sect. 2) and sector bounded, i.e., there exist numbers $a \leq b$ such that

$$
\langle\phi(v)-a v, \phi(v)-b v\rangle \leq 0, \quad \forall v \in U
$$

Let $\mathcal{S}[a, b]$ denote the set of all functions $\phi: U \rightarrow U$ such that (3.2) holds. It is easy to show that (3.2) holds if and only if

$$
\left\|\phi(v)-\frac{a+b}{2} v\right\| \leq \frac{b-a}{2}\|v\|, \quad \forall v \in U .
$$

This shows in particular, that if $\phi \in \mathcal{S}[a, b]$, then the graph of $\phi$ is contained in a (non-convex) cone with vertex at the origin. For $a<b$ we define

$$
\mathcal{S}[a, b):=\bigcup_{a \leq c<b} \mathcal{S}[a, c] .
$$

\section{Stability in the large}

The zero solution of (3.1) is called stable in the large if: (i) for all $\left(x^{0}, u^{0}\right) \in X \times U$ there exists a solution of (3.1) on $\mathbb{R}_{+}$(i.e. $T=\infty$ in Lem. 2.4); and (ii) there exists a continuous, strictly increasing function $p: \mathbb{R}_{+} \rightarrow \mathbb{R}_{+}$ with $p(0)=0$ and such that for any $l>0$ the solution of $(x(\cdot), u(\cdot))$ of the initial value (3.1) satisfies

$$
\left\|x^{0}\right\|+\left\|u^{0}\right\| \leq l \quad \Longrightarrow \quad\|x(t)\|+\|u(t)\| \leq p(l), \forall t \geq 0 .
$$


Let $R, S \in \mathcal{B}\left(U_{c}\right)$. If

$$
\langle R v, v\rangle \geq 0, \quad \forall v \in U_{c},
$$

we write $R \geq 0$; if the above inequality is strict for all $u \in U_{c} \backslash\{0\}$, we write $R>0$. Since $U_{c}$ is a complex Hilbert space, $R \geq 0$ implies that $R=R^{*}$. We write $S \geq R$ if $S-R \geq 0$.

The following theorem shows that a suitable positive real condition in terms of the transfer function $\mathbf{G}(s) / s$ will ensure that the zero solution of (3.1) is stable in the large if $\boldsymbol{\Sigma}$ is exponentially stable and $\phi \in \mathcal{S}[0, b)$.

Theorem 3.1. Suppose that $\boldsymbol{\Sigma}$ is exponentially stable, $\mathbf{G}(0)$ is invertible in $\mathcal{B}(U)$ and $\phi: U \rightarrow U$ is locally Lipschitz. Let $\left(x^{0}, u^{0}\right) \in X \times U$. If there exists $b>0$ such that

$$
I+\frac{b}{2}\left(\frac{1}{s} \mathbf{G}(s)+\frac{1}{\bar{s}} \mathbf{G}^{*}(s)\right) \geq 0, \quad \forall s \in \mathbb{C}_{0},
$$

and if $\phi \in \mathcal{S}[0, b)$, then the following statements hold:

(a) the zero solution of (3.1) is stable in the large;

(b) the solution $(x(\cdot), u(\cdot))$ of (3.1) satisfies

$$
\lim _{t \rightarrow \infty}\|x(t)\|=0, \quad x \in L^{2}\left(\mathbb{R}_{+}, X\right), \quad \lim _{t \rightarrow \infty}\|\phi(u(t))\|=0, \quad \phi \circ u \in L^{2}\left(\mathbb{R}_{+}, U\right) ;
$$

(c) under the extra assumption that $\operatorname{dim} U=1, u^{\infty}:=\lim _{t \rightarrow \infty} u(t)$ exists, is finite and satisfies $\phi\left(u^{\infty}\right)=0$.

Remark 3.2. (a) Since $\boldsymbol{\Sigma}$ is exponentially stable, it follows that $\mathbf{G}(s) / s$ is holomorphic in $\mathbb{C}_{0}$. Combining this with (PR) shows that the function $I+b \mathbf{G}(s) / s$ is positive real. As in the finite-dimensional case (see Anderson and Vongpanitlerd [2], p. 53), it can be shown that (PR) holds if and only if $\mathbf{G}(0)=\mathbf{G}^{*}(0) \geq 0$ and

$$
I+\frac{b}{2}\left(\frac{1}{i \omega} \mathbf{G}(i \omega)-\frac{1}{i \omega} \mathbf{G}^{*}(i \omega)\right) \geq 0, \quad \forall \omega \in \mathbb{R} \backslash\{0\} .
$$

In this context it is interesting to note that there exists $b>0$ such that $(\mathrm{PR})$ holds if and only if $\mathbf{G}(0)=$ $\mathbf{G}^{*}(0) \geq 0$ (see Logemann and Townley [21]).

(b) Note that assertion (a) implies the boundedness of the solution $(x(\cdot), u(\cdot))$ of $(3.1)$ for all $\left(x^{0}, u^{0}\right) \in X \times U$.

(c) If $\phi^{-1}(\{0\})=\{0\}$ and $\operatorname{dim} U=1$, then it follows from a combination of assertions (a)-(c) that the zero solution of (3.1) is globally asymptotically stable.

(d) Some of the statements in Theorem 3.1 remain true for time-varying sector bounded nonlinearities. More precisely, let $\phi: \mathbb{R}_{+} \times U \rightarrow U,(t, v) \mapsto \phi(t, v)$ be continuous in $t$ and locally Lipschitz in $v$, uniformly in $t$ on bounded intervals. An inspection of the proof of Theorem 3.1 shows that statement (a) remains true for all such $\phi$ satisfying

$$
\langle\phi(t, v), \phi(t, v)-c v\rangle \leq 0, \quad \forall(t, v) \in \mathbb{R}_{+} \times U
$$

for some $c \in[0, b)$; furthermore, apart from the convergence of $\phi(t, u(t))$ to 0 as $t \rightarrow \infty$, statement (b) remains true also.

(e) Consider the feedback system shown in Figure 3, where $\mathbf{H}(s)$ is the (rational) transfer function of a $m$-input, $m$-output, exponentially stable, finite-dimensional system and $\phi: \mathbb{R}^{m} \rightarrow \mathbb{R}^{m}$ is a static nonlinearity. The circle criterion says that if

$$
I+\frac{b}{2}\left(\mathbf{H}(i \omega)+\mathbf{H}^{*}(i \omega)\right) \geq 0, \quad \forall \omega \in \mathbb{R}
$$

then for all locally Lipschitz $\phi \in \mathcal{S}[0, b)$, the closed-loop system shown in Figure 3 is globally exponentially stable (see [13], p. 409 and [28], p. 227). Hence Theorem 3.1 can be considered as an extension of the circle criterion to the case where $\mathbf{H}(s)$ is of the form $\mathbf{H}(s)=\mathbf{G}(s) / s$ with $\mathbf{G}$ being the transfer function of an exponentially stable well-posed infinite-dimensional system, i.e. the plant is the series interconnection of an exponentially stable well-posed infinite-dimensional system and an integrator. 


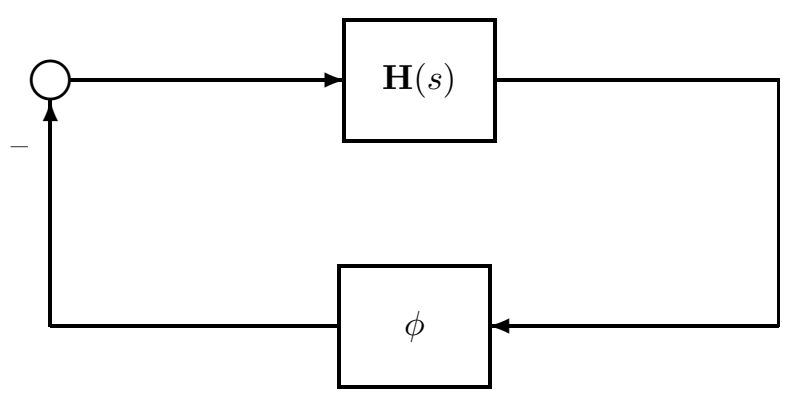

Figure 3

(f) For exponentially stable single-input single-output systems with bounded control and bounded observation, the stability properties of the zero solution of (3.1) have been investigated in [33] under the assumption that $\phi$ is locally Lipschitz, $\phi(v) v>0$ for $v \neq 0$ and $\lim _{|v| \rightarrow \infty} \int_{0}^{v} \phi(w) d w=\infty$. It is shown in [33] that for all such $\phi$, the zero solution of (3.1) is uniformly asymptotically stable in the large, provided that

$$
\operatorname{Re} \mathbf{G}(i \omega) \geq \varepsilon>0, \quad \forall \omega \in \mathbb{R},
$$

i.e., $\mathbf{G}$ is positive-real in a strict sense ${ }^{4}$. Trivially, there are many examples where (3.3) is not satisfied, whilst (PR) holds for some $b>0$.

Proof of Theorem 3.1: Let $\left(x^{0}, u^{0}\right) \in X \times U$. By Lemma 2.4 the corresponding solution of the initial-value problem $(3.1)$, denoted by $(x(\cdot), u(\cdot))$, exists on a maximal interval $[0, T)$ (where $0<T \leq \infty)$ and is unique. Set $Q:=\mathbf{G}(0)$ and define for $t \in[0, T)$

$$
z(t):=A^{-1} x(t), \quad v(t):=Q^{-1}(C z(t)+u(t))
$$

We proceed in several steps.

Step 1: A differential equation for $(z, v)$.

We claim that $(z(t), v(t))$ is continuously differentiable in $X \times U$ for all $t \in(0, T)$ and satisfies

$$
\begin{aligned}
& \dot{z}(t)=A z(t)+A^{-1} B \phi(u(t)), \quad z(0)=z^{0} \in X_{1}, \quad \forall t \in(0, T), \\
& \dot{v}(t)=-\phi(u(t)), \quad v(0)=v^{0} \in U, \quad \forall t \in(0, T)
\end{aligned}
$$

where $z^{0}:=A^{-1} x^{0}$ and $v^{0}:=Q^{-1}\left(C A^{-1} x^{0}+u^{0}\right)$.

Using the continuity of the functions $t \mapsto \phi(u(t))$ and

$$
[0, T) \rightarrow X, t \mapsto \int_{0}^{t} \mathbf{T}_{t-\tau} B \phi(u(\tau)) d \tau
$$

it follows from a well-known result on the existence of classical solutions to abstract Cauchy problems (see Pazy [22], p. 107) that for all $t \in(0, T), x(t)$ is continuously differentiable in $X_{-1}$ and (3.1a) holds. As a consequence, $z(t)$ is continuously differentiable in $X$ for all $t \in(0, T)$ and $(3.5 \mathrm{a})$ holds. The proof of the claim for $v(t)$ is more difficult and requires an approximation argument. To this end let $T_{0} \in(0, T)$ be arbitrary.

\footnotetext{
${ }^{4}$ If the underlying semigroup is analytic, then this result remains true for unbounded control action, see [4].
} 
Choose $x_{n}^{0} \in X_{1}$ and $w_{n} \in W^{1,2}\left(\left[0, T_{0}\right], U\right)$ with $w_{n}(0)=0$ such that

$$
\lim _{n \rightarrow \infty}\left\|x^{0}-x_{n}^{0}\right\|=0, \quad \lim _{n \rightarrow \infty}\left\|\phi(u)-w_{n}\right\|_{L^{2}(0, T ; U)}=0 .
$$

Consider the initial-value problem

$$
\begin{aligned}
& \dot{\zeta}(t)=A \zeta(t)+B w_{n}(t), \quad \zeta(0)=x_{n}^{0}, \\
& \dot{\eta}(t)=-C \mathbf{T}_{t} x_{n}^{0}-\left(\mathbf{F}_{\infty} w_{n}\right)(t), \quad \eta(0)=u^{0},
\end{aligned}
$$

and denote its solution by $\left(x_{n}(\cdot), u_{n}(\cdot)\right)$. Denoting the output function of $\boldsymbol{\Sigma}$ corresponding to the initial value $x_{n}^{0}$ and the control $w_{n}(\cdot)$ by $y_{n}(\cdot)$, it is clear that the right-hand side of $(3.7 \mathrm{~b})$ is equal to $-y_{n}(t)$. By Lemma $2.3, x_{n}$ is continuously differentiable in $X$ and hence the function defined by

$$
v_{n}(t):=Q^{-1}\left[C A^{-1} x_{n}(t)+u_{n}(t)\right]
$$

is absolutely continuous with derivative

$$
\begin{aligned}
\dot{v}_{n}(t) & =Q^{-1}\left(C\left[x_{n}(t)+A^{-1} B w_{n}(t)\right]-y_{n}(t)\right) \\
& =Q^{-1}\left(C\left[\mathbf{T}_{t} x_{n}^{0}+\int_{0}^{t} \mathbf{T}_{t-\tau} B w_{n}(\tau) d \tau+A^{-1} B w_{n}(t)\right]-y_{n}(t)\right), \quad \text { a.e. } t \in\left[0, T_{0}\right] .
\end{aligned}
$$

Invoking (2.7), we obtain

$$
\dot{v}_{n}(t)=-Q^{-1} \mathbf{G}(0) w_{n}(t)=-w_{n}(t), \quad \text { a.e. } t \in\left[0, T_{0}\right]
$$

and thus

$$
v_{n}(t)=v_{n}(0)-\int_{0}^{t} w_{n}(\tau) d \tau, \quad \forall t \in\left[0, T_{0}\right] .
$$

From (3.6) it follows via standard properties of well-posed systems that for all $t \in\left[0, T_{0}\right]$

$$
\lim _{n \rightarrow \infty}\left\|x_{n}(t)-x(t)\right\|=0, \quad \lim _{n \rightarrow \infty} u_{n}(t)=u(t),
$$

and therefore, by (3.4) and (3.8)

On the other hand, by (3.6) and (3.9)

$$
\lim _{n \rightarrow \infty} v_{n}(t)=v(t), \quad \forall t \in\left[0, T_{0}\right] .
$$

$$
\lim _{n \rightarrow \infty} v_{n}(t)=v^{0}-\int_{0}^{t} \phi(u(\tau)) d \tau, \quad \forall t \in\left[0, T_{0}\right],
$$

showing that

which upon differentiation yields (3.5b).

$$
v(t)=v^{0}-\int_{0}^{t} \phi(u(\tau)) d \tau, \quad \forall t \in\left[0, T_{0}\right],
$$

Step 2: Exploiting the positive-real condition (PR).

By assumption, $\phi \in \mathcal{S}[0, b)$, and hence there exists $\tilde{b} \in(0, b)$ such that

$$
\phi \in \mathcal{S}[0, \tilde{b}] .
$$


Choose $c \in(\tilde{b}, b)$. We consider the quadruple $\boldsymbol{\Xi}=\left(A, A^{-1} B, C, c^{-1} I\right)$ of operators, which are the generating operators of an exponentially stable Pritchard-Salamon system ${ }^{5}$ on the spaces $X_{1} \hookrightarrow X$ (this implies that $\boldsymbol{\Xi}$ defines an exponentially stable regular system). The function

$$
\mathbf{H}(s)=C(s I-A)^{-1} A^{-1} B+\frac{1}{c} I
$$

is the transfer function of $\boldsymbol{\Xi}$. By (2.9) we have

$$
\mathbf{H}(s)=\frac{1}{s}(\mathbf{G}(s)-\mathbf{G}(0))+\frac{1}{c} I=\frac{1}{s}(\mathbf{G}(s)-Q)+\frac{1}{c} I, \quad \forall s \in \varrho(A), s \neq 0 .
$$

It is not difficult to show that (PR) implies that $Q=\mathbf{G}(0) \geq 0$, and hence, in particular, $Q=Q^{*}$ (this can be proved exactly as in the finite-dimensional case, see [2], p. 53; cf. also Rem. 3.2, Part (a)). Therefore, since $0<c<b$, (PR) guarantees the existence of a constant $\varepsilon>0$ such that

$$
\mathbf{H}(i \omega)+\mathbf{H}^{*}(i \omega) \geq \varepsilon I, \quad \forall w \in \mathbb{R} .
$$

Consequently, by the positive-real Riccati equation theory for Pritchard-Salamon systems in van Keulen [16] (see Th. 3.10 and Rem. 3.14 in [16]), there exists $\tilde{P} \in \mathcal{B}(X), \tilde{P}=\tilde{P}^{*}$, such that

$$
\left\langle A x_{1}, \tilde{P} x_{2}\right\rangle+\left\langle\tilde{P} x_{1}, A x_{2}\right\rangle=\frac{c}{2}\left\langle\left[\left(A^{-1} B\right)^{*} \tilde{P}+C\right] x_{1},\left[\left(A^{-1} B\right)^{*} \tilde{P}+C\right] x_{2}\right\rangle, \quad \forall x_{1}, x_{2} \in X_{1} .
$$

Setting

we obtain using (3.11)

$$
P:=-\tilde{P} \in \mathcal{B}(X), \quad L:=\sqrt{\frac{c}{2}}\left[C-\left(A^{-1} B\right)^{*} P\right] \in \mathcal{B}\left(X_{1}, U\right),
$$

$$
\begin{aligned}
\left\langle A x_{1}, P x_{2}\right\rangle+\left\langle P x_{1}, A x_{2}\right\rangle & =-\left\langle L x_{1}, L x_{2}\right\rangle, \quad \forall x_{1}, x_{2} \in X_{1} \\
\left(A^{-1} B\right)^{*} P x_{1} & =C x_{1}-\sqrt{2 / c} L x_{1}, \quad \forall x_{1} \in X_{1} .
\end{aligned}
$$

Moreover, by a routine argument it follows from (3.12a) and the exponential stability of the semigroup $\mathbf{T}_{t}$ that $P \geq 0$. Note that the existence of solutions $L$ and $P=P^{*} \geq 0$ to the Lure equations (3.12) is the content of the Kalman-Yakubovich lemma in the context of the infinite-dimensional system $\boldsymbol{\Xi}$.

Step 3: A Lyapunov-type argument.

For $t \in[0, T)$ define

$$
V(t):=\langle z(t), P z(t)\rangle+\langle v(t), Q v(t)\rangle \geq 0 .
$$

By Step $1, V$ is continuously differentiable. Differentiating $V$ and using $(3.5,3.12)$ and the definition of $v$ (see (3.4)) yields for all $t \in[0, T)$

$$
\begin{aligned}
\dot{V}(t) & =-\|L z(t)\|^{2}+2\langle\phi(u(t)), C z(t)-\sqrt{2 / c} L z(t)\rangle-2\langle\phi(u(t)), Q v(t)\rangle \\
& =-\|L z(t)\|^{2}-2 \sqrt{2 / c}\langle\phi(u(t)), L z(t)\rangle-2\langle\phi(u(t)), u(t)\rangle .
\end{aligned}
$$

Completing the square gives for all $t \in[0, T)$

$$
\dot{V}(t)=-\|L z(t)+\sqrt{2 / c} \phi(u(t))\|^{2}+\frac{2}{c}\left(\|\phi(u(t))\|^{2}-c\langle\phi(u(t)), u(t)\rangle\right) .
$$

\footnotetext{
${ }^{5}$ See $[9,16]$ for the concept of a Pritchard-Salamon system.
} 
Since $c>\tilde{b}$ there exists $\delta>0$ such that $c=\tilde{b}+\delta$ and therefore, using (3.10), we obtain for all $t \in[0, T)$

$$
\begin{aligned}
\|\phi(u(t))\|^{2}-c\langle\phi(u(t)), u(t)\rangle & =\langle\phi(u(t)), \phi(u(t))-\tilde{b} u(t)\rangle-\delta\langle\phi(u(t)), u(t)\rangle \\
& \leq-\delta\langle\phi(u(t)), u(t)\rangle \leq-\frac{\delta}{\tilde{b}}\|\phi(u(t))\|^{2}
\end{aligned}
$$

Using this inequality in (3.14), it follows that

$$
\dot{V}(t) \leq-\frac{2 \delta}{\tilde{b} c}\|\phi(u(t))\|^{2}, \quad \forall t \in[0, T) .
$$

Hence, since $V(t) \geq 0$, integration leads to

$$
\int_{0}^{t}\|\phi(u(\tau))\|^{2} d \tau \leq \frac{\tilde{b} c}{2 \delta} V(0), \quad \forall t \in[0, T) .
$$

The inequality (3.15) shows that $\phi \circ u \in L^{2}(0, T ; U)$. Combining this with the well-posedness and the exponential stability of $\boldsymbol{\Sigma}$, we may conclude that $x(t)$ is bounded on the interval $[0, T)$ and $\dot{u} \in L^{2}(0, T ; U)$. This implies the boundedness of $(x(t), u(t))$ on $[0, T)$ if $T<\infty$, and hence, by the maximality of $T$, it follows via Lemma 2.4 that $T=\infty$. Therefore the solution $(x(t), u(t))$ exists for all $t \geq 0$ and by $(3.15)$

$$
\int_{0}^{\infty}\|\phi(u(\tau))\|^{2} d \tau \leq \frac{\tilde{b} c}{2 \delta} V(0) .
$$

Step 4: Proof of statement (a) - stability in the large.

As an immediate consequence of the exponential stability of $\boldsymbol{\Sigma},(2.3,3.4,3.13)$ and $(3.16)$ there exist constants $\alpha_{1}, \alpha_{2}, \alpha_{3}>0$ not depending on $x^{0}$ and $u^{0}$ and such that

$$
\|x(t)\|^{2} \leq \alpha_{1}\left(\left\|x^{0}\right\|^{2}+V(0)\right) \leq \alpha_{2}\left(\left\|x^{0}\right\|^{2}+\left\|A^{-1} x^{0}\right\|^{2}+\left\|C A^{-1} x^{0}+u^{0}\right\|^{2}\right) \leq \alpha_{3}\left(\left\|x^{0}\right\|^{2}+\left\|u^{0}\right\|^{2}\right) .
$$

Moreover, as was pointed out in Step 2, (PR) implies that $Q=Q^{*} \geq 0$. Combining this with the invertibility of $Q$, it follows from standard results on positive operators (see e.g. Rudin [23], p. 314) that the map $w \mapsto$ $\langle w, Q w\rangle^{1 / 2}$ defines a new norm on $U$ which is equivalent to the original norm on $U$. Therefore, using (3.13), the fact that $V(t)$ is non-increasing and $P \geq 0$, we may conclude that

$$
\|v(t)\|^{2} \leq \beta_{1} V(0) \leq \beta_{2}\left(\left\|x^{0}\right\|^{2}+\left\|u^{0}\right\|^{2}\right)
$$

for some constants $\beta_{1}, \beta_{2}>0$ which do not depend on $x^{0}$ and $u^{0}$. From the definition of $v(t)($ see $(3.4))$ it follows that

$$
\left\|Q^{-1} u(t)\right\|^{2} \leq 2 \beta_{2}\left(\left\|x^{0}\right\|^{2}+\left\|u^{0}\right\|^{2}\right)+2\left\|Q^{-1} C A^{-1} x(t)\right\|^{2} .
$$

Combining this with (3.17) yields the existence of a constant $\beta_{3}>0$ (not depending on $x^{0}$ and $u^{0}$ ) such that

$$
\|u(t)\|^{2} \leq \beta_{3}\left(\left\|x^{0}\right\|^{2}+\left\|u^{0}\right\|^{2}\right) .
$$

Statement (a) now follows from (3.17) and (3.18).

Step 5: Proof of statement (b) - asymptotic behaviour of $x$ and $\phi \circ u$.

Note that by (3.16),

$$
\phi \circ u \in L^{2}\left(\mathbb{R}_{+}, U\right)
$$


and hence, by Lemma $2.2, x \in L^{2}\left(\mathbb{R}_{+}, X\right)$ and $\lim _{t \rightarrow \infty}\|x(t)\|=0$. It only remains to show that $\lim _{t \rightarrow \infty} \phi(u(t))=$ 0 . To this end note, that that by statement (a) proved in Step $4, u(\cdot)$ is bounded, i.e. there exists a number $\gamma>0$ such that

$$
\|u(t)\| \leq \gamma, \quad \forall t \geq 0
$$

Moreover, by the exponential stability of $\boldsymbol{\Sigma}$ we obtain from $(3.1 \mathrm{~b})$ and (3.19) that $\dot{u} \in L^{2}\left(\mathbb{R}_{+}, U\right)$, which implies in particular that $u(\cdot)$ is uniformly continuous. By (3.20) and the local Lipschitz property of $\phi$, the restricted $\left.\operatorname{map} \phi\right|_{\operatorname{im} u}$ is globally Lipschitz, and therefore, using the uniform continuity of $u(\cdot)$, we may conclude that $\phi \circ u$ is uniformly continuous. Combining this with (3.19) and applying Barbălat's lemma (see [13], p. 192) shows that $\lim _{t \rightarrow \infty} \phi(u(t))=0$.

Step 6: Proof of statement (c) - asymptotic behaviour of $u$.

Assume that $\operatorname{dim} U=1$, i.e. $U=\mathbb{R}$. Note, that by Step $3, V_{\infty}:=\lim _{t \rightarrow \infty} V(t)$ exists and is finite $($ since $V(\cdot)$ is non-negative and non-increasing). Combining this with the fact that $\lim _{t \rightarrow \infty}\|x(t)\|=0$, we may conclude from (3.4) and (3.13) that $\lim _{t \rightarrow \infty} u(t)=u^{\infty}$ exists and is finite. By continuity of $\phi$ and statement (b), it follows that $\phi\left(u^{\infty}\right)=\lim _{t \rightarrow \infty} \phi(u(t))=0$.

In the following we introduce extra assumptions on the nonlinearity $\phi$ which will guarantee that $u(t)$ converges as $t \rightarrow \infty$ in the case that $\operatorname{dim} U>1$.

Corollary 3.3. Suppose that the assumptions of Theorem 3.1 hold. If there exists $b>0$ such that (PR) is satisfied, if $\phi \in \mathcal{S}[0, b)$ and if the extra assumptions

(A1) $\phi^{-1}(\{0\}) \cap W$ is finite for any bounded set $W \subset U$,

(A2) $\inf _{w \in W}\|\phi(w)\|>0$ for any bounded, closed and nonempty set $W \subset U$ with $\phi^{-1}(\{0\}) \cap W=\emptyset$,

are satisfied, then statements (a) and (b) of Theorem 3.1 hold. Moreover, the limit $u^{\infty}:=\lim _{t \rightarrow \infty} u(t)$ exists, is finite and $\phi\left(u^{\infty}\right)=0$.

Remark 3.4. Of course, assumption (A2) is automatically satisfied if $\operatorname{dim} U<\infty$. Corollary 3.3 shows in particular that if $\phi^{-1}(\{0\})=\{0\}$ and (A2) holds, then the zero solution of (3.1) is globally asymptotically stable.

Proof of Corollary 3.3: Let $(x(\cdot), u(\cdot))$ denote the solution of (3.1). Clearly, since the assumptions of Theorem 3.1 are satisfied, statements (a) and (b) of Theorem 3.1 hold. Therefore, in particular, im $u$ is bounded and

$$
\lim _{t \rightarrow \infty} \phi(u(t))=0
$$

By assumption (A1), the set $Z:=\phi^{-1}(\{0\}) \cap \operatorname{clos}(\operatorname{im} u)$ is finite. Moreover, by (A2) and (3.21), $Z \neq \emptyset$. Note that since $(x(\cdot), u(\cdot))$ is the solution of $(3.1), u(\cdot)$ is (absolutely) continuous. So it is sufficient to show that

$$
\lim _{t \rightarrow \infty} \operatorname{dist}(u(t), Z)=0
$$

since by finiteness of $Z$ and continuity of $u,(3.22)$ implies that for all sufficiently large $t, u(t)$ lies in a neighbourhood of exactly one point of $Z$ and hence $u_{\infty}:=\lim _{t \rightarrow \infty} u(t)$ exists with $u_{\infty} \in Z \subset \phi^{-1}(\{0\})$.

Seeking a contradiction, suppose that (3.22) is not true. Then there exist a sequence $\left(t_{n}\right) \subset \mathbb{R}_{+}$with $\lim _{n \rightarrow \infty} t_{n}=\infty$ and a number $\varepsilon>0$ such that

$$
u\left(t_{n}\right) \notin\{v \in U: \operatorname{dist}(v, Z)<\varepsilon\}=: Z_{\varepsilon} .
$$

Defining $W:=\operatorname{clos}\left(\operatorname{im} u \backslash Z_{\varepsilon}\right)$, it follows that $W$ is closed, bounded and $\phi^{-1}(\{0\}) \cap W=\emptyset$. Consequently, by assumption (A2), there exists $\delta>0$ such that $\|\phi(w)\| \geq \delta$ for all $w \in W$. Since $u\left(t_{n}\right) \in W$ for all $n \in \mathbb{N}$, we obtain that $\left\|\phi\left(u\left(t_{n}\right)\right)\right\| \geq \delta$ for all $n \in \mathbb{N}$, contradicting (3.21). 


\section{Exponential stability}

The following theorem shows that under the assumptions of Theorem 3.1 the zero solution of (3.1) is globally exponentially stable, provided that $\phi \in \mathcal{S}[a, b)$ for some $a \in(0, b)$ (i.e., the nonlinearity $\phi$ is assumed to satisfy a more restrictive sector condition than in Th. 3.1).

Theorem 3.5. Suppose that $\mathbf{\Sigma}$ is exponentially stable, $\mathbf{G}(0)$ is invertible and $\phi: U \rightarrow U$ is locally Lipschitz. If there exists $b>0$ such that (PR) holds and if $\phi \in \mathcal{S}[a, b)$ for some $a \in(0, b)$, then the zero solution of (3.1) is globally exponentially stable, that is, there exist $N \geq 1$ and $\nu \in(\omega(\mathbf{T}), 0)$ such that for all $\left(x^{0}, u^{0}\right) \in X \times U$ the solution $(x(\cdot), u(\cdot))$ of (3.1) satisfies

$$
\|x(t)\|+\|u(t)\| \leq N e^{\nu t}\left(\left\|x^{0}\right\|+\left\|u^{0}\right\|\right), \quad \forall t \geq 0 .
$$

Proof: Let $K$ be the supremum of all numbers $k>0$ such that

$$
I+\frac{k}{2}\left(\frac{1}{s} \mathbf{G}(s)+\frac{1}{\bar{s}} \mathbf{G}^{*}(s)\right) \geq 0, \quad \forall s \in \mathbb{C}_{0} .
$$

By assumption, $K \geq b>0$. Since $\phi \in \mathcal{S}[a, b)$, there exists $c \in(a, b)$ such that $\phi \in \mathcal{S}[a, c]$. Let $\delta \in(0, a)$ such that $c+\delta<K$. We define

$$
\kappa:=\frac{c+\delta}{2}<\frac{K}{2}, \quad \mathbf{H}(s):=\frac{1}{s} \mathbf{G}(s)\left(I+\frac{\kappa}{s} \mathbf{G}(s)\right)^{-1}, \quad \mathbf{L}(s):=\frac{1}{s}\left(I+\frac{\kappa}{s} \mathbf{G}(s)\right)^{-1} .
$$

We know from $[21]^{6}$ that

$$
\mathbf{H}, \mathbf{L} \in H^{\infty}\left(\mathbb{C}_{0}, \mathcal{B}(U)\right) .
$$

Moreover, Lemma 3.10 in [21] yields

$$
\|\mathbf{H}\|_{\infty}=\sup _{s \in \mathbb{C}_{0}}\|\mathbf{H}(s)\|=\frac{1}{\kappa} .
$$

Setting

and using the fact that $\phi \in \mathcal{S}[a, c] \subset \mathcal{S}[\delta, c]$, we obtain

$$
\psi(v):=\phi(v)-\kappa v, \quad \gamma:=\frac{c-\delta}{2}
$$

$$
\|\psi(v)\| \leq \gamma\|v\|, \quad \forall v \in U .
$$

Clearly, $\kappa>\gamma$, and hence by $(3.24)$

$$
\gamma\|\mathbf{H}\|_{\infty}<1
$$

Let $\varepsilon \in(0, \delta)$ be sufficiently small such that the semigroup $e^{\varepsilon t} \mathbf{T}_{t}$ is exponentially stable,

$$
\mathbf{H}, \mathbf{L} \in H^{\infty}\left(\mathbb{C}_{-\varepsilon}, \mathcal{B}(U)\right),
$$

and

$$
\gamma \sup _{s \in \mathbb{C}_{-\varepsilon}}\|\mathbf{H}(s)\|<1
$$

\footnotetext{
${ }^{6}$ The results in [21] are proved for matrix-valued $\mathbf{G}$, i.e. $\operatorname{dim} U<\infty$. However, it is easy to see that the relevant results in [21] extend to infinite-dimensional input spaces $U$.
} 
For all sufficiently small $\varepsilon>0,(3.27)$ follows via a routine argument from (3.23) and the fact that $\mathbf{G} \in$ $H^{\infty}\left(\mathbb{C}_{-\varepsilon}, \mathcal{B}(U)\right)$, whilst $(3.28)$ is a consequence of $(3.26)$ and (3.27) combined with the fact that a holomorphic function which is bounded in an open vertical strip in the complex plane is uniformly continuous in any closed vertical substrip (see [8], p. 72).

Let $\left(x^{0}, u^{0}\right) \in X \times U$ and let $(x(\cdot), u(\cdot))$ denote the solution of (3.1) which satisfies the initial conditions $x(0)=x^{0}$ and $u(0)=u^{0}$. Rewrite $(3.1 \mathrm{~b})$ in the form

$$
\dot{u}(t)=-\left(\boldsymbol{\Psi}_{\infty} x^{0}\right)(t)-\left(\mathbf{F}_{\infty}(\psi \circ u+\kappa u)\right)(t), \quad \text { a.e. } t \geq 0 .
$$

By Theorem 3.1, $u$ is bounded and $\dot{u} \in L^{2}\left(\mathbb{R}_{+}, U\right)$. Thus the Laplace transforms $(\mathfrak{L}(u))(s),(\mathfrak{L}(\psi \circ u))(s)$ and $(\mathfrak{L}(\dot{u}))(s)$ exist for all $s \in \mathbb{C}_{0}$ and an application of the Laplace transform to (3.29) combined with a straightforward calculation yields

$$
(\mathfrak{L}(u))(s)=\mathbf{L}(s) u^{0}-\mathbf{L}(s) C(s I-A)^{-1} x^{0}-\mathbf{H}(s)(\mathfrak{L}(\psi \circ u))(s), \quad \forall s \in \mathbb{C}_{0} .
$$

Define bounded operators $H, L$ from $L^{2}\left(\mathbb{R}_{+}, U\right)$ to $L^{2}\left(\mathbb{R}_{+}, U\right)$ by setting

$$
H v=\mathfrak{L}^{-1}(\mathbf{H} \mathfrak{L}(v)), \quad L v=\mathfrak{L}^{-1}(\mathbf{L} \mathfrak{L}(v)) ; \quad \forall v \in L^{2}\left(\mathbb{R}_{+}, U\right)
$$

By $(3.27), H$ and $L$ restrict to bounded operators from $L_{-\varepsilon}^{2}\left(\mathbb{R}_{+}, U\right)$ to $L_{-\varepsilon}^{2}\left(\mathbb{R}_{+}, U\right)$. The $L_{-\varepsilon}^{2}\left(\mathbb{R}_{+}, U\right)$-induced operator norms of $H$ and $L$ are given by

$$
\sup _{s \in \mathbb{C}_{-\varepsilon}}\|\mathbf{H}(s)\|=: h \quad \text { and } \sup _{s \in \mathbb{C}_{-\varepsilon}}\|\mathbf{L}(s)\|=: l,
$$

respectively. Taking inverse Laplace transforms in (3.30), we obtain

$$
u=\mathfrak{L}^{-1}\left(\mathbf{L} u^{0}\right)-L\left(\Psi_{\infty} x^{0}\right)-H(\psi \circ u) .
$$

Taking the $L_{-\varepsilon}^{2}$-norm of $\mathbf{P}_{t} u$ (where $t \geq 0$ ), using the causality of $H$ and estimating gives

$$
\begin{aligned}
\left(\int_{0}^{t}\left\|e^{\varepsilon \tau} u(\tau)\right\|^{2} d \tau\right)^{1 / 2} & \leq\left(\int_{0}^{\infty}\left\|e^{\varepsilon \tau}\left(\mathfrak{L}^{-1}\left(\mathbf{L} u^{0}\right)\right)(\tau)\right\|^{2} d \tau\right)^{1 / 2}+l\left(\int_{0}^{\infty}\left\|e^{\varepsilon \tau} C_{L} \mathbf{T}_{\tau} x^{0}\right\|^{2} d \tau\right)^{1 / 2} \\
& +h\left(\int_{0}^{t}\left\|e^{\varepsilon \tau} \psi(u(\tau))\right\|^{2} d \tau\right)^{1 / 2}, \quad \forall t \geq 0
\end{aligned}
$$

It is clear that the function $s \mapsto \mathbf{L}(s) u^{0}$ is in $H^{2}\left(\mathbb{C}_{-\varepsilon}, U\right)$, and therefore by a well-known theorem due to Paley and Wiener

$$
\int_{0}^{\infty}\left\|e^{\varepsilon \tau}\left(\mathfrak{L}^{-1}\left(\mathbf{L} u^{0}\right)\right)(\tau)\right\|^{2} d \tau=\frac{1}{2 \pi} \sup _{\alpha>-\varepsilon} \int_{-\infty}^{\infty}\left\|\mathbf{L}(\alpha+i \omega) u^{0}\right\|^{2} d \omega \leq N_{1}^{2}\left\|u^{0}\right\|^{2}
$$

where

$$
N_{1}:=\left(\frac{1}{2 \pi} \sup _{\alpha>-\varepsilon} \int_{-\infty}^{\infty}\|\mathbf{L}(\alpha+i \omega)\|^{2} d \omega\right)^{1 / 2}<\infty
$$

Combining $(3.25,3.32,3.33)$ and using the exponential stability of $e^{\varepsilon t} \mathbf{T}_{t}$, we may conclude that there exists $N_{2}>0$ (not depending on $x^{0}$ and $u^{0}$ ) such that

$$
\left(\int_{0}^{t}\left\|e^{\varepsilon \tau} u(\tau)\right\|^{2} d \tau\right)^{1 / 2} \leq N_{1}\left\|u^{0}\right\|+N_{2}\left\|x^{0}\right\|+\gamma h\left(\int_{0}^{t}\left\|e^{\varepsilon \tau} u(\tau)\right\|^{2} d \tau\right)^{1 / 2}, \quad \forall t \geq 0 .
$$


By (3.28) and (3.31), $\gamma h<1$, and therefore, $u \in L_{-\varepsilon}^{2}\left(\mathbb{R}_{+}, U\right)$, and, moreover,

$$
\left(\int_{0}^{\infty}\left\|e^{\varepsilon \tau} u(\tau)\right\|^{2} d \tau\right)^{1 / 2} \leq N_{3}\left(\left\|x^{0}\right\|+\left\|u^{0}\right\|\right)
$$

where

$$
N_{3}:=\frac{\max \left(N_{1}, N_{2}\right)}{1-\gamma h}
$$

Furthermore, since $\mathbf{F}_{\infty}$ is bounded from $L_{-\varepsilon}^{2}\left(\mathbb{R}_{+}, U\right)$ to $L_{-\varepsilon}^{2}\left(\mathbb{R}_{+}, U\right)$ with $L_{-\varepsilon}^{2}$-induced operator norm equal to

$$
f_{\infty}:=\sup _{s \in \mathbb{C}_{-\varepsilon}}\|\mathbf{G}(s)\|
$$

and $\phi \in \mathcal{S}[a, c]$, it follows from (3.34) that

$$
\left(\int_{0}^{\infty}\left\|e^{\varepsilon \tau}\left(\mathbf{F}_{\infty} \phi(u)\right)(\tau)\right\|^{2} d \tau\right)^{1 / 2} \leq N_{3} f_{\infty} c\left(\left\|x^{0}\right\|+\left\|u^{0}\right\|\right) .
$$

In order to obtain an exponential estimate for $x(t)$, we multiply the integrated version of (3.1a) by $e^{\varepsilon t}$ to obtain

$$
e^{\varepsilon t} x(t)=e^{\varepsilon t} \mathbf{T}_{t} x^{0}+\int_{0}^{t} e^{\varepsilon(t-\tau)} \mathbf{T}_{t-\tau} B e^{\varepsilon \tau} \phi(u(\tau)) d \tau, \quad \forall t \geq 0 .
$$

Using the exponential stability of the semigroup $e^{\varepsilon t} \mathbf{T}_{t}$ and the fact that $\phi \in \mathcal{S}[a, b)$, it follows that there exists a constant $N_{4} \geq 1$ (not depending on $x^{0}$ and $u^{0}$ ) such that

$$
e^{\varepsilon t}\|x(t)\| \leq N_{4}\left[\left\|x^{0}\right\|+\left(\int_{0}^{t}\left\|e^{\varepsilon \tau} u(\tau)\right\|^{2} d \tau\right)^{1 / 2}\right], \quad \forall t \geq 0 .
$$

Combining this with (3.34) shows that

$$
e^{\varepsilon t}\|x(t)\| \leq N_{5}\left(\left\|x^{0}\right\|+\left\|u^{0}\right\|\right), \quad \forall t \geq 0
$$

for some $N_{5} \geq 1$ (not depending on $x^{0}$ and $u^{0}$ ).

Finally, to derive an exponential estimate for $u(t)$, let $\eta \in(0, \varepsilon)$. Using (3.1b), a straightforward calculation gives

$$
\frac{d}{d t}\left(e^{\eta t} u(t)\right)=-e^{(\eta-\varepsilon) t} C_{L} e^{\varepsilon t} \mathbf{T}_{t} x^{0}-e^{(\eta-\varepsilon) t}\left[e^{\varepsilon t}\left(\mathbf{F}_{\infty} \phi(u)\right)(t)\right]+\eta e^{(\eta-\varepsilon) t}\left[e^{\varepsilon t} u(t)\right], \quad \text { a.e. } t \geq 0 .
$$

Clearly, the functions

$$
t \mapsto e^{(\eta-\varepsilon) t}, t \mapsto C_{L} e^{\varepsilon t} \mathbf{T}_{t} x^{0}
$$

are in $L^{2}\left(\mathbb{R}_{+}, \mathbb{R}\right)$ and $L^{2}\left(\mathbb{R}_{+}, U\right)$, respectively. Moreover, by (3.34) and (3.35) the functions

$$
t \mapsto e^{\varepsilon t} u(t), t \mapsto e^{\varepsilon t}\left(\mathbf{F}_{\infty} \phi(u)\right)(t)
$$

are in $L^{2}\left(\mathbb{R}_{+}, U\right)$. Integrating (3.37), using the Cauchy-Schwarz inequality and invoking (3.34) and (3.35) shows that there exists a constant $N_{6} \geq 1$ (not depending on $x^{0}$ and $u^{0}$ ) such that

$$
e^{\eta t}\|u(t)\| \leq N_{6}\left(\left\|x^{0}\right\|+\left\|u^{0}\right\|\right), \quad \forall t \geq 0 .
$$


The claim now follows from (3.36) and (3.38) with $N=2 \max \left(N_{5}, N_{6}\right)$ and $\nu=-\eta$.

For $W \subset U$ and $a \leq b$, let $\mathcal{S}_{W}[a, b]$ denote the set of all functions $\phi: U \rightarrow U$ such that

$$
\langle\phi(w)-a w, \phi(w)-b w\rangle \leq 0, \quad \forall w \in W .
$$

For $a<b$ we define

$$
\mathcal{S}_{W}[a, b):=\bigcup_{a \leq c<b} \mathcal{S}_{W}[a, c] .
$$

Of course, $\mathcal{S}_{U}[a, b]=\mathcal{S}[a, b]$ and $\mathcal{S}_{U}[a, b)=\mathcal{S}[a, b)$.

Theorem 3.1 and Theorem 3.5 can be used to derive the following semi-global exponential stability result. The assumptions on the nonlinearity $\phi$ are more restrictive than in Theorem 3.1, but less restrictive than in Theorem 3.5.

Theorem 3.6. Suppose that $\boldsymbol{\Sigma}$ is exponentially stable, $\mathbf{G}(0)$ is invertible, $\phi: U \rightarrow U$ is locally Lipschitz and there exists $b>0$ such that $(P R)$ holds. If $\phi \in \mathcal{S}[0, b)$ and $\phi$ satisfies the two extra assumptions

(A3) $\phi \in \mathcal{S}_{V}[a, b)$ for some open set $V \subset U$ with $0 \in V$ and some $a \in(0, b)$,

(A4) $\inf _{w \in W}\|\phi(w)\|>0$ for any bounded, closed, nonempty set $W \subset U$ with $0 \notin W$,

then the zero solution of (3.1) is semi-globally exponentially stable, that is, for every $M>0$, there exists $N \geq 1$ and $\nu \in(\omega(\mathbf{T}), 0)$ such that for all $\left(x^{0}, u^{0}\right) \in X \times U$ with $\left\|x^{0}\right\|+\left\|u^{0}\right\| \leq M$, the solution $(x(\cdot), u(\cdot))$ of (3.1) satisfies

$$
\|x(t)\|+\|u(t)\| \leq N e^{\nu t}\left(\left\|x^{0}\right\|+\left\|u^{0}\right\|\right), \quad \forall t \geq 0 .
$$

Of course, for finite-dimensional $U$, (A4) holds if $\phi^{-1}(\{0\})=\{0\}$ (by the continuity of $\phi$ ).

Proof of Theorem 3.6: Let $M>0$. By statement (a) of Theorem 3.1, there exists $R>0$ such that for all $\left(x^{0}, u^{0}\right) \in X \times U$ with $\left\|x^{0}\right\|+\left\|u^{0}\right\| \leq M$, the solution $(x(\cdot), u(\cdot))$ of (3.1) satisfies

$$
\|x(t)\|+\|u(t)\| \leq R, \quad \forall t \geq 0 .
$$

Since $\phi \in \mathcal{S}[0, b)$ and by assumption (A3), there exists $c \in(a, b)$ such that

$$
\phi \in \mathcal{S}[0, c] \cap \mathcal{S}_{V}[a, c] .
$$

Define $\psi: U \rightarrow U$ by

$$
\psi(v)=\left\{\begin{array}{lll}
\phi(v) & \text { if } & \|v\| \leq R \\
c\left(1-\frac{R}{\|v\|}\right) v+\phi\left(\frac{R}{\|v\|} v\right) & \text { if } & \|v\|>R
\end{array} .\right.
$$

Clearly, $\psi$ is locally Lipschitz. Moreover, by Corollary A.2 in Part 1 of the Appendix, there exists $\varepsilon>0$ such that $\psi \in \mathcal{S}[\varepsilon, b)$ (this is evident in the case $\operatorname{dim} U=1$ ). Consequently, we may apply Theorem 3.5 to the system

$$
\begin{aligned}
& \dot{\zeta}(t)=A \zeta(t)+B \psi(\eta(t)), \quad \zeta(0)=\zeta^{0}, \\
& \dot{\eta}(t)=-C_{L} \mathbf{T}_{t} x^{0}-\left[\mathbf{F}_{\infty}(\psi(\eta))\right](t), \quad \eta(0)=\eta^{0},
\end{aligned}
$$

to conclude that the zero solution of (3.41) is globally exponentially stable. By (3.39) and (3.40) it is clear that for all initial conditions $\left(x^{0}, u^{0}\right) \in X \times U$ with $\left\|x^{0}\right\|+\left\|u^{0}\right\| \leq M$, the solution $(x(\cdot), u(\cdot))$ of $(3.1)$ is also 
a solution of (3.41). Therefore, by Theorem 3.5, there exists $N \geq 1$ and $\nu \in(\omega(\mathbf{T}), 0)$ such that for all initial conditions $\left(x^{0}, u^{0}\right) \in X \times U$ with $\left\|x^{0}\right\|+\left\|u^{0}\right\| \leq M$,

$$
\|x(t)\|+\|u(t)\| \leq N e^{\nu t}\left(\left\|x^{0}\right\|+\left\|u^{0}\right\|\right), \quad \forall t \geq 0 .
$$

\section{Applications to LOW-Gain integral CONTrol}

In this section we apply the results in Section 3 to the so-called low-gain tracking problem described in the Introduction (see also Fig. 2). As in Section 3, let $\boldsymbol{\Sigma}=(\mathbf{T}, \boldsymbol{\Phi}, \boldsymbol{\Psi}, \mathbf{F})$ be a well-posed linear system with state space $X$, input space $U$, output space $Y=U$, generating operators $A, B$ and $C$, input-output operator $\mathbf{F}_{\infty}$ and transfer function $\mathbf{G}$. In this section we assume that $U=Y=\mathbb{R}^{m}$.

For $\lambda>0$, let $\mathcal{N}_{1}(\lambda)$ denote the set of all nondecreasing globally Lipschitz nonlinearities $f: \mathbb{R} \rightarrow \mathbb{R}$ such that $\lambda$ is a Lipschitz constant for $f$. Note that if $f \in \mathcal{N}_{1}(\lambda)$ and $f(0)=0$, then $f \in \mathcal{S}[0, \lambda]$. Moreover, for $\lambda>0$, $\mathcal{N}_{m}(\lambda)$ denotes the set of all nonlinearities $f: \mathbb{R}^{m} \rightarrow \mathbb{R}^{m}$ which are of the form

$$
f(v)=\left[f_{1}\left(v_{1}\right), f_{2}\left(v_{2}\right), \ldots, f_{m}\left(v_{m}\right)\right]^{T}, \quad \forall v=\left(v_{1}, v_{2}, \ldots, v_{m}\right)^{T} \in \mathbb{R}^{m},
$$

where $f_{i} \in \mathcal{N}_{1}(\lambda)$ for all $i=1, \ldots, m$. Sometimes it will be convenient to write (4.1) in the form $f=\operatorname{diag}\left(f_{i}\right)$. Clearly, a nonlinearity in $\mathcal{N}_{m}(\lambda)$ is globally Lipschitz.

Consider the following nonlinear system

$$
\begin{aligned}
& \dot{x}(t)=A x(t)+B \phi(u(t)), \quad x(0)=x^{0} \in X \\
& \dot{u}(t)=k\left\{r-C_{L} \mathbf{T}_{t} x^{0}-\left[\mathbf{F}_{\infty}(\phi(u))\right](t)\right\}, \quad u(0)=u^{0} \in \mathbb{R}^{m},
\end{aligned}
$$

where $r \in \mathbb{R}^{m}$ is the reference vector, $k \in \mathbb{R}$ is a gain parameter and $\phi: \mathbb{R}^{m} \rightarrow \mathbb{R}^{m}$ is a nonlinearity in $\mathcal{N}_{m}(\lambda)$. The aim is to show that under suitable conditions on $\boldsymbol{\Sigma}$, the error $e(t)=r-y(t)$, where $y(t)=$ $C_{L} \mathbf{T}_{t} x^{0}+\left[\mathbf{F}_{\infty}(\phi(u))\right](t)$, becomes small in some sense as $t \rightarrow \infty$.

If $\mathbf{G}$ is holomorphic and bounded on $\mathbb{C}_{-\varepsilon}$ for some $\varepsilon>0$ (which for example is the case if $\boldsymbol{\Sigma}$ is exponentially stable) and $\mathbf{G}(0)=\mathbf{G}^{*}(0)>0$, then it is not difficult to show that the following positive-real condition

$$
I+\frac{k}{2}\left(\frac{1}{s} \mathbf{G}(s)+\frac{1}{\bar{s}} \mathbf{G}^{*}(s)\right) \geq 0, \quad \forall s \in \mathbb{C}_{0}
$$

holds for all sufficiently small $k>0$, see Lemma 3.10 in [21]. We define

$$
K:=\sup \{k>0:(4.3) \text { holds }\}
$$

Recall that $\mathcal{M}$ denotes the space of all $\mathbb{R}^{m \times m}$-valued Borel measures on $\mathbb{R}_{+}$.

Theorem 4.1. Assume that $\boldsymbol{\Sigma}$ is exponentially stable and $\mathbf{G}(0)=\mathbf{G}^{*}(0)>0$. Let $\lambda>0, \phi \in \mathcal{N}_{m}(\lambda)$, $k \in(0, K / \lambda)$ and let $r \in \mathbb{R}^{m}$ be such that

$$
\phi^{r}:=[\mathbf{G}(0)]^{-1} r \in \operatorname{im} \phi .
$$

Then the solution $(x(\cdot), u(\cdot))$ of (4.2) is unique and exists on $\mathbb{R}_{+}$, and for each $u^{r} \in \phi^{-1}\left(\left\{\phi^{r}\right\}\right)$, there exists $N>0$ such that for all $\left(x^{0}, u^{0}\right) \in X \times \mathbb{R}^{m}$

$$
\left\|x(t)-x^{r}\right\|+\left\|u(t)-u^{r}\right\| \leq N\left(\left\|x^{0}-x^{r}\right\|+\left\|u^{0}-u^{r}\right\|\right), \quad \forall t \geq 0,
$$

where $x^{r}:=-A^{-1} B \phi^{r}$. Moreover, the following statements hold: 
(a) $\lim _{t \rightarrow \infty} \phi(u(t))=\phi^{r}, \quad \phi \circ u-\phi^{r} \in L^{2}\left(\mathbb{R}_{+}, \mathbb{R}^{m}\right)$;

(b) $\lim _{t \rightarrow \infty}\left\|x(t)-x^{r}\right\|=0, \quad x-x^{r} \in L^{2}\left(\mathbb{R}_{+}, X\right)$;

(c) $e:=r-y \in L^{2}\left(\mathbb{R}_{+}, \mathbb{R}^{m}\right)$, where $y(t)=C_{L} \mathbf{T}_{t} x^{0}+\left[\mathbf{F}_{\infty}(\phi(u))\right](t)$;

(d) under the additional assumption that $m=1$, the limit $\lim _{t \rightarrow \infty} u(t)=: u^{\infty}$ exists, is finite and satisfies $\phi\left(u^{\infty}\right)=\phi^{r}$

(e) under the additional assumption that $\phi^{-1}\left(\left\{\phi^{r}\right\}\right) \cap W$ is finite for any bounded set $W \subset \mathbb{R}^{m}$, the limit $\lim _{t \rightarrow \infty} u(t)=: u^{\infty}$ exists, is finite and satisfies $\phi\left(u^{\infty}\right)=\phi^{r}$;

(f) under the additional assumption that $\mathfrak{L}^{-1}(\mathbf{G}) \in \mathcal{M}$, the error e satisfies $e=e_{1}+e_{2}$, where $e_{1}$ is a bounded function with $\lim _{t \rightarrow \infty} e_{1}(t)=0$ and $e_{2} \in L_{\alpha}^{2}\left(\mathbb{R}_{+}, \mathbb{R}^{m}\right)$ for any $\alpha>\omega(\mathbf{T})$; if additionally $\mathbf{T}_{t^{0}} x^{0} \in X_{1}$ for some $t^{0} \geq 0$, then $\lim _{t \rightarrow \infty} e_{2}(t)=0$.

Remark 4.2. (a) Whilst statement (c) of Theorem 4.1 need not imply asymptotic tracking, it does imply that the error is small for large $t$ in the sense that for all $\delta, \varepsilon>0$, there exists $\tau>0$ such that

$$
\mu_{L}(\{t \geq \tau:\|e(t)\| \geq \delta\}) \leq \varepsilon,
$$

where $\mu_{L}$ denotes the Lebesgue measure on $\mathbb{R}_{+}$.

(b) The assumption in statement (f) of Theorem 4.1 that $\mathfrak{L}^{-1}(\mathbf{G}) \in \mathcal{M}$ implies the regularity of $\boldsymbol{\Sigma}$. However, this assumption is not very restrictive in the sense that it seems to be satisfied in all practical examples of exponentially stable well-posed systems. In particular, it is satisfied if $B$ or $C$ is bounded (see Lem. 2.3 in [18]). Statement (f) implies that $\lim _{t \rightarrow \infty} e(t)=0$, provided that $\mathfrak{L}^{-1}(\mathbf{G}) \in \mathcal{M}$ and $\mathbf{T}_{t^{0}} x^{0} \in X_{1}$ for some $t^{0} \geq 0$.

(c) In applying Theorem 4.1 it is important to know the constant $K$ or at least a lower bound for $K$. In the single-input, single-output case it has been shown in [19] how $K$ can be obtained from frequency-response experiments performed on the linear part of the plant.

(d) In the multivariable case, the applicability of Theorem 4.1 is severely limited by the assumption $\mathbf{G}(0)=$ $\mathbf{G}^{*}(0)>0$. If we relax this hypothesis on $\mathbf{G}(0)$ and only assume that $\operatorname{det} \mathbf{G}(0) \neq 0$, then there exists $\Gamma \in \mathbb{R}^{m \times m}$ such that $\Gamma \mathbf{G}(0)=\mathbf{G}^{*}(0) \Gamma^{*}>0$ (choose, for example, $\Gamma=[\mathbf{G}(0)]^{-1}$ ). If the control law (4.2b) is replaced by

$$
\dot{u}(t)=k \Gamma\left\{r-C_{L} \mathbf{T}_{t} x^{0}-\left[\mathbf{F}_{\infty}(\phi(u))\right](t)\right\}
$$

and $\mathbf{G}(s)$ is replaced by $\Gamma \mathbf{G}(s)$ in (4.3), then the conclusions of Theorem 4.1 remain true. However, finding a suitable "matrix gain" $\Gamma$ will usually require exact knowledge of $\mathbf{G}(0)$.

(e) We remark that Theorem 4.1 considerably improves the main result of [20] (see Th. 3.3 in [20]) in the sense that Theorem 4.1 (i) guarantees better asymptotic and faster convergence properties and (ii) is not restricted to single-input single-output regular systems, but applies to the wider class of multivariable well-posed systems. In particular, the following parts of Theorem 4.1 are new: the stability property (4.5), the fact that $\phi \circ u-\phi^{r} \in L^{2}\left(\mathbb{R}_{+}, \mathbb{R}^{m}\right)$ and $x-x^{r} \in L^{2}\left(\mathbb{R}_{+}, X\right)$ and statements (c)-(e).

Proof of Theorem 4.1: Let $\left(x^{0}, u^{0}\right) \in X \times U$. By Lemma 2.4, the corresponding solution of the initial-value problem (4.2), denoted by $(x(\cdot), u(\cdot))$, is unique and, since $\phi$ is globally Lipschitz, it exists on $\mathbb{R}_{+}$. Let $u^{r} \in$ $\phi^{-1}\left(\left\{\phi^{r}\right\}\right)$ and introduce the nonlinearity

$$
\psi: \mathbb{R}^{m} \rightarrow \mathbb{R}^{m}, \quad w \mapsto \phi\left(w+u^{r}\right)-\phi^{r} .
$$

Since $\phi \in \mathcal{N}_{m}(\lambda)$, it is straightforward to show that $\psi \in \mathcal{S}[0, \lambda]$. Set

$$
z(\cdot):=x(\cdot)-x^{r}, \quad v(\cdot):=u(\cdot)-u^{r} .
$$


We proceed in two steps.

Step 1: We claim that

$$
\begin{aligned}
& \dot{z}(t)=A z(t)+B \psi(v(t)), \quad z(0)=z^{0}:=x^{0}-x^{r} \in X, \\
& \dot{v}(t)=-k\left\{C_{L} \mathbf{T}_{t} z^{0}+\left[\mathbf{F}_{\infty}(\psi(v))\right](t)\right\}, \quad v(0)=v^{0}:=u^{0}-u^{r} \in U,
\end{aligned}
$$

where, as usual, the derivative on the left-hand side of (4.8a) has to be interpreted in $X_{-1}$. Equation (4.8a) follows easily from (4.2a). Moreover, setting

$$
\tilde{v}(t):=r-C_{L} \mathbf{T}_{t} x^{r}-\left[\mathbf{F}_{\infty}\left(\phi^{r}\right)\right](t),
$$

we obtain from $(4.2 \mathrm{~b})$ that

$$
\dot{v}(t)=k\left\{\tilde{v}(t)-C_{L} \mathbf{T}_{t} z^{0}-\left[\mathbf{F}_{\infty}(\psi(v))\right](t)\right\} .
$$

It remains to show that $\tilde{v}(t)=0$ for a.e. $t \geq 0$. Using Laplace transforms and (2.9), we obtain

$$
\begin{aligned}
(\mathfrak{L}(\tilde{v}))(s) & =\frac{r}{s}-C(s I-A)^{-1} x^{r}-\frac{1}{s} \mathbf{G}(s) \phi^{r} \\
& =-\frac{1}{s}(\mathbf{G}(s)-\mathbf{G}(0)) \phi^{r}+C(s I-A)^{-1} A^{-1} B \phi^{r}=0
\end{aligned}
$$

showing that $\tilde{v}(t)=0$ for a.e. $t \geq 0$.

Step 2: For $k \in(0, K / \lambda)$, we may apply Theorem 3.1 to (4.8) in order to derive (4.5) and

$$
\lim _{t \rightarrow \infty}\|z(t)\|=0, \quad z \in L^{2}\left(\mathbb{R}_{+}, X\right), \quad \lim _{t \rightarrow \infty} \psi(v(t))=0, \quad \psi \circ v \in L^{2}\left(\mathbb{R}_{+}, \mathbb{R}^{m}\right) .
$$

Statements (a) and (b) now follow from (4.9). Furthermore, using that $\phi^{-1}\left(\left\{\phi^{r}\right\}\right)=\psi^{-1}(\{0\})+u^{r}$, statements (d) and (e) are easy consequences of Theorem 3.1, Corollary 3.3 and Remark 3.4. Statement (c) follows from Lemma 2.1, the exponential stability of $\boldsymbol{\Sigma}$ and statement (a). To prove statement (f), decompose $e=e_{1}+e_{2}$, where

$$
e_{1}(t):=r-\left[\mathfrak{L}^{-1}(\mathbf{G}) \star \phi(u)\right](t), \quad e_{2}(t):=-C_{L} \mathbf{T}_{t} x^{0}
$$

Clearly, using the exponential stability of $\mathbf{T}, e_{2} \in L_{\alpha}^{2}\left(\mathbb{R}_{+}, \mathbb{R}^{m}\right)$ for any $\alpha>\omega(\mathbf{T})$ and $\lim _{t \rightarrow \infty} e_{2}(t)=0$ if $\mathbf{T}_{t^{0}} x^{0} \in X_{1}$ for some $t^{0} \geq 0$. Finally, since $\lim _{t \rightarrow \infty} \phi(u(t))=\phi^{r}$ and $\mathfrak{L}^{-1}(\mathbf{G}) \in \mathcal{M}$, it follows from Gripenberg et al. [11] (Th. 6.1, Part (ii), p. 96) that

$$
\lim _{t \rightarrow \infty} e_{1}(t)=r-\mathbf{G}(0) \phi^{r}=r-r=0 .
$$

Remark 4.3. Suppose that $\phi=\operatorname{diag}\left(\phi_{i}\right)$, where the functions $\phi_{i}: \mathbb{R} \rightarrow \mathbb{R}$ are non-decreasing. If we replace the global Lipschitz assumption on $\phi_{i}$ by the weaker assumption that $\phi_{i}$ is locally Lipschitz and $\phi_{i}(\cdot)-\phi_{i}(0) \in \mathcal{S}[0, b]$ for some $b \geq 0$, then it is not difficult to prove that the function $\psi$ defined in (4.6) is in $\mathcal{S}[0, \tilde{b}]$ for some $\tilde{b} \geq 0$. An inspection of the proof of Theorem 4.1 then shows that the conclusions of Theorem 4.1 remain true for all sufficiently small $k>0$.

Under certain additional assumptions on $\phi$ and $r$, it can be shown that the variables $x, u$ and $y$ converge exponentially fast. This will be addressed in the next result, Theorem 4.5. In order to state and prove this theorem, we need some preparation. 

by

Let $f \in \mathcal{N}_{1}(\lambda)$. The Clarke $[5,6]$ generalized directional derivative $f^{\circ}(v ; w)$ of $f$ at $v$ in direction $w$ is given

$$
f^{\circ}(v ; w)=\limsup _{\substack{\xi \rightarrow v \\ h \downarrow 0}} \frac{f(\xi+h w)-f(\xi)}{h} .
$$

Define $f^{-}(\cdot):=-f^{\circ}(\cdot ;-1)$ (if $f$ is $C^{1}$ with derivative $f^{\prime}$, then $\left.f^{-} \equiv f^{\prime}\right)$. A point $v \in \mathbb{R}$ is said to be a critical point (and $f(v)$ is said to be a critical value) of $f$ if $f^{-}(v)=0$. A point $v=\left(v_{1}, \ldots, v_{m}\right)^{T} \in \mathbb{R}^{m}$ is called a critical point (and $f(v)$ is said to be a critical value) of $f=\operatorname{diag}\left(f_{i}\right) \in \mathcal{N}_{m}(\lambda)$ if there exists $j \in\{1, \ldots, m\}$ such that $v_{j}$ is a critical point of $f_{j}$. Note that if $f \in \mathcal{N}_{m}(\lambda)$ and $w \in \operatorname{im} f$ is not a critical value of $f$, then $f^{-1}(\{w\})$ is a singleton.

We record the following technicality for later use. The proof can be found in the Appendix, Part 2.

Lemma 4.4. Let $\lambda>0$ and $f \in \mathcal{N}_{m}(\lambda)$. If $f(0)=0$ and 0 is not a critical point of $f$, then there exist $a \in(0, \lambda)$ and an open set $V \subset \mathbb{R}^{m}$ with $0 \in V$ such that $f \in \mathcal{S}_{V}[a, \lambda]$.

Recall that by definition a $\mathbb{R}^{m \times m}$-valued Borel measure $\mu$ on $\mathbb{R}_{+}$is in $\mathcal{M}_{\alpha}$ (where $\alpha \in \mathbb{R}$ ) if the exponentially weighted measure $E \mapsto \int_{E} e^{-\alpha t} \mu(d t)$ belongs to $\mathcal{M}$. Equivalently, $\mathcal{M}_{\alpha}$ is the space of all $\mathbb{R}^{m \times m}$-valued Borel measures $\mu$ on $\mathbb{R}_{+}$such that $\int_{0}^{\infty} e^{-\alpha t}|\mu|(d t)<\infty$, where $|\mu|$ denotes the total variation of $\mu$.

Theorem 4.5. Assume that $\boldsymbol{\Sigma}$ is exponentially stable and $\mathbf{G}(0)=\mathbf{G}^{*}(0)>0$. Let $\lambda>0, \phi \in \mathcal{N}_{m}(\lambda)$ and $k \in(0, K / \lambda)$. Let $r \in \mathbb{R}^{m}$ be such that

$$
\phi^{r}:=[\mathbf{G}(0)]^{-1} r \in \operatorname{im} \phi
$$

and $\phi^{r}$ is not a critical value of $\phi$. Let $u^{r}$ be the unique element in $\mathbb{R}^{m}$ such that $\phi\left(u^{r}\right)=\phi^{r}$ and set $x^{r}:=$ $-A^{-1} B \phi^{r}$. Then, for given $M>0$, there exist $N \geq 1$ and $\nu \in(\omega(\mathbf{T}), 0)$ such that for all $\left(x^{0}, u^{0}\right) \in X \times \mathbb{R}^{m}$ with $\left\|x^{0}-x^{r}\right\|+\left\|u^{0}-u^{r}\right\| \leq M$, the solution $(x(\cdot), u(\cdot))$ of (4.2) satisfies

$$
\left\|x(t)-x^{r}\right\|+\left\|u(t)-u^{r}\right\| \leq N e^{\nu t}\left(\left\|x^{0}-x^{r}\right\|+\left\|u^{0}-u^{r}\right\|\right), \quad \forall t \geq 0,
$$

and

$$
\left\|\phi(u(t))-\phi^{r}\right\| \leq \lambda N e^{\nu t}\left(\left\|x^{0}-x^{r}\right\|+\left\|u^{0}-u^{r}\right\|\right), \quad \forall t \geq 0 .
$$

Moreover, for all $\left(x^{0}, u^{0}\right) \in X \times \mathbb{R}^{m}$ with $\left\|x^{0}-x^{r}\right\|+\left\|u^{0}-u^{r}\right\| \leq M$, the following statements hold:

(a) for any $\alpha>\nu, e:=r-y \in L_{\alpha}^{2}\left(\mathbb{R}_{+}, \mathbb{R}^{m}\right)$, where $y(t)=C_{L} \mathbf{T}_{t} x^{0}+\left[\mathbf{F}_{\infty}(\phi(u))\right](t)$;

(b) under the additional assumption that $\mathfrak{L}^{-1}(\mathbf{G}) \in \mathcal{M}_{\alpha}$ for some $\alpha<0$, the error e satisfies $e=e_{1}+e_{2}$, where $e_{1} \in L_{\beta}^{\infty}\left(\mathbb{R}_{+}, \mathbb{R}^{m}\right)$ for any $\beta \geq \max (\alpha, \nu)$, and $e_{2} \in L_{\beta}^{2}\left(\mathbb{R}_{+}, \mathbb{R}^{m}\right)$ for any $\beta>\omega(\mathbf{T})$; if additionally $\mathbf{T}_{t^{0}} x^{0} \in X_{1}$ for some $t^{0} \geq 0$, then $e_{2} \in L_{\beta}^{\infty}\left(\mathbb{R}_{+}, \mathbb{R}^{m}\right)$ for any $\beta>\omega(\mathbf{T})$.

Remark 4.6. (a) Statement (b) shows that exponentially fast asymptotic tracking is guaranteed if $\mathfrak{L}^{-1}(\mathbf{G}) \in$ $\mathcal{M}_{\alpha}$ for some $\alpha<0$ and $\mathbf{T}_{t^{0}} x^{0} \in X_{1}$ for some $t^{0} \geq 0$. Again, the assumption that $\mathfrak{L}^{-1}(\mathbf{G}) \in \mathcal{M}_{\alpha}$ for some $\alpha<0$ is not very restrictive and seems to be satisfied in all practical examples of exponentially stable well-posed systems. In particular, this assumption is satisfied if $B$ or $C$ is bounded (see Lem. 2.3 in [18]).

(b) We mention that Parts (c) and (d) of Remark 4.2 and Remark 4.3 remain relevant in the context of Theorem 4.5.

(c) As compared to the main result in [20] (see Th. 3.3 in [20]), Theorem 4.5 is entirely new: the issue of exponential decay is not addressed in [20].

Proof of Theorem 4.5: Let $\left(x^{0}, u^{0}\right) \in X \times U$. By Lemma 2.4, the corresponding solution of the initial-value problem (4.2), denoted by $(x(\cdot), u(\cdot))$, is unique and exists on $\mathbb{R}_{+}$. Let $\psi, z$ and $v$ be defined by (4.6) and (4.7). Clearly, by the assumptions on $\phi, \psi \in \mathcal{N}_{m}(\lambda), \psi^{-1}(\{0\})=\{0\}$ and 0 is not a critical value of $\psi$. Invoking Lemma 4.4, we see that there exists $a \in(0, \lambda)$ and an open set $V \subset \mathbb{R}^{m}$ with $0 \in V$ such that $\psi \in \mathcal{S}_{V}[a, \lambda]$. For $k \in(0, K / \lambda)$ we may apply Theorem 3.6 to (4.8) in order to derive (4.11) and (4.12). Statement (a) follows 
from Lemma 2.1 and (4.12). In order to prove statement (b), we write $e=e_{1}+e_{2}$, with $e_{1}$ and $e_{2}$ defined by (4.10). The claim for $e_{2}$ follows immediately from standard results on admissible observation operators. Finally, to prove exponential convergence of $e_{1}$, set $\mu:=\mathfrak{L}^{-1}(\mathbf{G})$. By assumption $\mu \in \mathcal{M}_{\alpha}$ for some $\alpha<0$. The function $t \mapsto\left\|e_{1}(t)\right\|$ can be estimated as follows

$$
\left\|e_{1}(t)\right\| \leq\left\|\left[\mu \star\left(\phi(u)-\phi^{r} \theta\right)(t)\right]\right\|+\left\|\left(\mu \star \phi^{r} \theta\right)(t)-\mathbf{G}(0) \phi^{r}\right\|, \quad \forall t \geq 0
$$

where $\theta$ denotes the unit-step (Heaviside) function. Let $\beta \geq \max (\alpha, \nu)$. Then, by (4.12), the function $t \mapsto$ $e^{-\beta t}\left\|\phi(u(t))-\phi^{r}\right\|$ remains bounded as $t \rightarrow \infty$. Since $\mu \in \mathcal{M}_{\alpha}$, the measure $E \mapsto \int_{E} e^{-\alpha t} \mu(d t)$ belongs to $\mathcal{M}$. Hence, by [11] (Th. 3.5, Part (i), p. 119), we may conclude that there exists $M_{1}>0$ such that

$$
e^{-\beta t}\left\|\left[\mu \star\left(\phi(u)-\phi^{r} \theta\right)\right](t)\right\| \leq M_{1}, \quad \forall t \geq 0 .
$$

Moreover, $M_{2}:=\int_{0}^{\infty} e^{-\alpha t}|\mu|(d t)<\infty$, and thus

$$
e^{-\beta t}\left\|\left(\mu \star \phi^{r} \theta\right)(t)-\mathbf{G}(0) \phi^{r}\right\| \leq\left\|\phi^{r}\right\| e^{-\alpha t} \int_{t}^{\infty}|\mu|(d \tau) \leq\left\|\phi^{r}\right\| \int_{0}^{\infty} e^{-\alpha \tau}|\mu|(d \tau)=M_{2}\left\|\phi^{r}\right\| .
$$

Consequently, appealing to (4.13-4.15), we deduce that the function $\mathbb{R}_{+} \rightarrow \mathbb{R}, t \mapsto e^{-\beta t}\left\|e_{1}(t)\right\|$ is bounded.

\section{Example: Diffusion process With output delay}

Consider a diffusion process (with diffusion coefficient $\kappa>0$ and with Dirichlet boundary conditions), on the one-dimensional spatial domain $I=(0,1)$, with scalar nonlinear pointwise control action (applied at point $x_{b} \in I$, via a nonlinearity $\phi$ with Lipschitz constant $\lambda>0$ ) and delayed (delay $h \geq 0$ ) pointwise scalar observation (at point $x_{c} \in I, x_{c}>x_{b}$.). We formally write this diffusion process as

$$
\begin{aligned}
& z_{t}(t, x)=\kappa z_{x x}(t, x)+\delta\left(x-x_{b}\right) \phi(u(t)), \quad y(t)=z\left(t-h, x_{c}\right) \\
& z(t, 0)=0=z(t, 1), \quad \text { for all } t>0 .
\end{aligned}
$$

For simplicity, we assume zero initial conditions:

$$
z(t, x)=0, \quad \text { for all }(t, x) \in[-h, 0] \times[0,1] .
$$

This system was analyzed in the context of low-gain integral control in $[19,20]$. With input $\phi(u(\cdot))$ and output $y(\cdot)$, this example qualifies as a well-posed linear system with transfer function given by

$$
\mathbf{G}(s)=\frac{e^{-s h} \sinh \left(x_{b} \sqrt{(s / \kappa)}\right) \sinh \left(\left(1-x_{c}\right) \sqrt{(s / \kappa)}\right)}{\kappa \sqrt{(s / \kappa)} \sinh \sqrt{(s / \kappa)}}
$$

It is not difficult to show that $\mathfrak{L}^{-1}(\mathbf{G}) \in L_{\alpha}^{1}\left(\mathbb{R}_{+}, \mathbb{R}\right) \subset \mathcal{M}_{\alpha}$ for any $\alpha>-\kappa \pi^{2}$.

From [19] we know that

$$
K=\sup \{k>0 \mid(4.3) \text { holds }\}=\frac{1}{\left|\mathbf{G}^{\prime}(0)\right|}=\frac{6 \kappa^{2}}{x_{b}\left(1-x_{c}\right)\left(6 h \kappa+1-x_{b}^{2}-\left(1-x_{c}\right)^{2}\right)} .
$$

Note the dependence of $K$ on the time-delay $h$ : the larger $h$, the smaller $K$. By Theorem 4.5, Part (b), for each $k \in(0, K / \lambda)$, the integral control

$$
u(t)=k \int_{0}^{t}[r-y(t)] d t
$$


guarantees exponentially fast asymptotic tracking of every constant reference value $r$ such that

$$
\phi^{r}=\frac{r}{\mathbf{G}(0)}=\frac{\kappa r}{x_{b}\left(1-x_{c}\right)} \in \operatorname{im} \phi
$$

and $\phi^{r}$ is not a critical value of $\phi$. Note that $\phi^{r}$ does not depend on $h$. It is easy to see that if $0 \in \operatorname{im} \phi$, then the range of values of $r$ which can be tracked becomes maximal if $x_{c} \downarrow x_{b}=1 / 2$.

For purposes of illustration, we adopt the following values

$$
\kappa=0.1, \quad x_{b}=\frac{1}{3}, \quad x_{c}=\frac{2}{3}, \quad h=1
$$

and we consider a nonlinearity $\phi$ of saturation type, defined as follows

$$
u \mapsto \phi(u):= \begin{cases}1, & u \geq 1 \\ u, & u \in(0,1) \\ 0, & u \leq 0\end{cases}
$$

In this case, $K=243 / 620 \approx 0.3919$ and $\lambda=1$. The critical values of $\phi$ are 0 and 1 . For $r=1$, we have

$$
\phi^{r}=\frac{r}{\mathbf{G}(0)}=\frac{\kappa}{x_{b}\left(1-x_{c}\right)}=0.9 \in[0,1]=\operatorname{im} \phi .
$$

In particular, $\phi^{r}$ is not a critical value of $\phi$. In each of the following three cases of controller gains

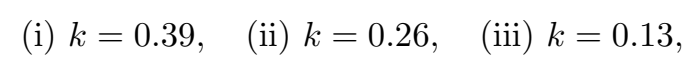

Figure 4 depicts the output behaviour of the system under integral control, while Figures 5 and 6 depict the corresponding control input and integrator state, respectively. Figure 7 illustrates the evolution of the temperature profile $z(t, \cdot)$ in case (i). These figures were generated using SIMULINK Simulation Software within MATLAB, wherein a truncated eigenfunction expansion, of order 20, was adopted to model the diffusion process.

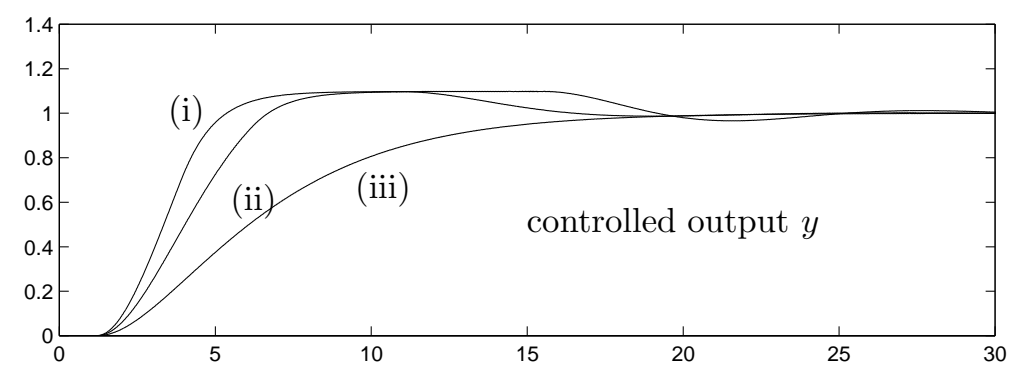

Figure 4: Controlled output. 


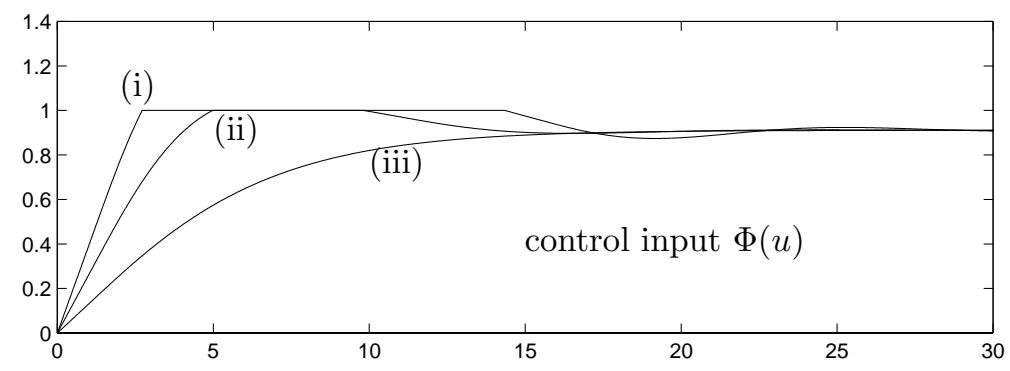

Figure 5: Control input.

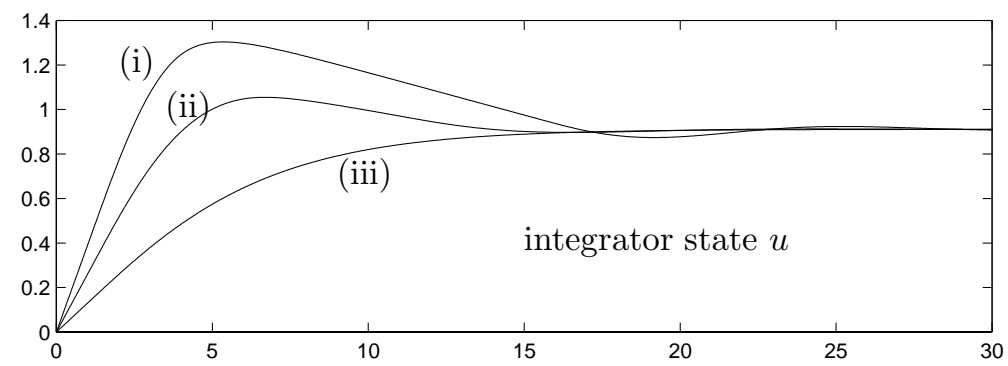

Figure 6: Integrator state.

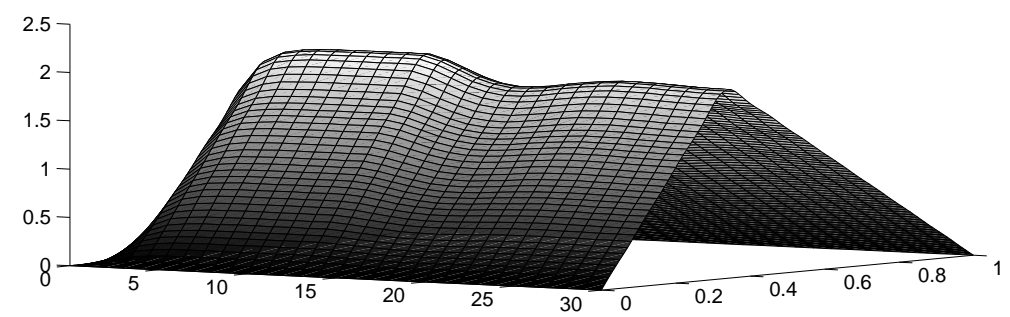

Figure 7: Temperature profile in case (i) $(k=0.39)$.

\section{Conclusions}

In this paper we have proved a number of absolute stability results for well-posed infinite-dimensional systems which guarantee, depending on the assumptions imposed on the nonlinearity, stability in the large, semi-global exponential stability or global exponential stability. These results are certainly new in the context of infinitedimensional systems, but might also exhibit some novelty in the finite-dimensional case: the authors were unable to find finite-dimensional versions of the main results in Section 3 in the literature. Our approach is based on a particular coordinate transformation combined with a Lyapunov-type analysis of the transformed system in which the positive-real Riccati equation theory for Pritchard-Salamon systems given in [16] plays an important role. In Section 4 the absolute stability results were applied to the low-gain integral control problem resulting in substantial improvements of the main result in [20]. An interesting problem for future research is the question whether the absolute stability results in Section 3 remain true if we replace the positive-real condition (PR) by 
the less restrictive assumption that there exists $q \geq 0$ such that

$$
\frac{2}{b} I+q\left(\mathbf{G}(s)+\mathbf{G}^{*}(s)\right)+\left(\frac{1}{s} \mathbf{G}(s)+\frac{1}{\bar{s}} \mathbf{G}^{*}(s)\right) \geq 0, \quad \forall s \in \mathbb{C}_{0} .
$$

This seems to be a difficult and challenging problem: in particular, a Lyapunov stability analysis based on the above positive-real condition (for $q>0$ ) would involve considerably more unboundedness than the Lyapunov analysis for the case $q=0$ given in Section 3 .

\section{APPENDiX}

\section{Part 1}

In this part of the Appendix we prove that there exists $\varepsilon>0$ such that the function $\psi: U \rightarrow U$ as defined in (3.40) is in $\mathcal{S}[\varepsilon, b)$, provided that $\phi \in \mathcal{S}[0, b)$ and $\phi$ satisfies assumptions (A3) and (A4) of Theorem 3.6. To this end we recall some properties of sector bounded nonlinearities which will be used freely in the following.

Let $W \subset U$ and $a \leq b$. For any function $f: U \rightarrow U$ we have

$$
f \in \mathcal{S}_{W}[a, b] \Longleftrightarrow\left\|f(w)-\frac{a+b}{2} w\right\| \leq \frac{b-a}{2}\|w\|, \forall w \in W .
$$

From this it follows that if $f \in \mathcal{S}_{W}[a, b]$, then $f \in \mathcal{S}_{W}[c, d]$ for all numbers $c$ and $d$ with $c \leq a \leq b \leq d$.

Lemma A.1. Let $f: U \rightarrow U$ be a function. Suppose that $f \in \mathcal{S}[0, b)$ for some $b>0$ and that

(a) there exists a neighbourhood $V \subset U$ of 0 and a number $a \in(0, b)$ such that $f \in \mathcal{S}_{V}[a, b)$;

(b) $\inf _{w \in W}\|f(w)\|>0$ for any bounded closed nonempty set $W \subset U$ with $0 \notin W$.

Under these conditions, for any bounded nonempty set $Y \subset U$, there exists $\varepsilon>0$ such that $f \in \mathcal{S}_{Y}[\varepsilon, b)$.

Of course, Lemma A.1 is not surprising and, if $\operatorname{dim} U=1$, the result is obvious and trivial. However, the case $\operatorname{dim} U>1$ requires a proof.

Proof of Lemma A.1: Let $Y \subset U$ be bounded and nonempty. Since $f \in \mathcal{S}[0, b)$ and by assumption (a), there exists $c \in(a, b)$ such that $f \in \mathcal{S}[0, c] \cap \mathcal{S}_{V}[a, c]$. Let $\eta>0$ be such that for all $v \in U$

$$
\|v\|<\eta \Rightarrow v \in V .
$$

Let $d \in(c, b)$. We claim that there exists $\varepsilon>0$ such that $f \in \mathcal{S}_{Y}[\varepsilon, d] \subset \mathcal{S}_{Y}[\varepsilon, b)$. Seeking a contradiction, suppose that the claim is not true. Then there exist sequences $\left(\varepsilon_{n}\right) \subset(0, a)$ with $\lim _{n \rightarrow \infty} \varepsilon_{n}=0$ and $\left(v_{n}\right) \subset Y$ such that

$$
\left\langle f\left(v_{n}\right)-\varepsilon_{n} v_{n}, f\left(v_{n}\right)-d v_{n}\right\rangle>0, \quad \forall n \in \mathbb{N} .
$$

Since $f \in \mathcal{S}_{V}[a, c] \subset \mathcal{S}_{V}\left[\varepsilon_{n}, d\right]$, we obtain from (A.2) and (A.3) that

$$
\left\|v_{n}\right\| \geq \eta>0, \quad \forall n \in \mathbb{N} .
$$

By (A.3),

$$
\left\|f\left(v_{n}\right)\right\|^{2} \geq\left(d+\varepsilon_{n}\right)\left\langle f\left(v_{n}\right), v_{n}\right\rangle-d \varepsilon_{n}\left\|v_{n}\right\|^{2}, \quad \forall n \in \mathbb{N} .
$$

Moreover, since $f \in \mathcal{S}[0, c]$

$$
\left\|f\left(v_{n}\right)\right\|^{2} \leq c\left\langle f\left(v_{n}\right), v_{n}\right\rangle, \quad \forall n \in \mathbb{N} .
$$


The set

$$
W:=\operatorname{clos}\left\{v_{n}: n \in \mathbb{N}\right\}
$$

is a bounded closed set, and, by (A.4), $0 \notin W$. By assumption (b), there exists $\nu_{1}>0$ such that

$$
\left\|f\left(v_{n}\right)\right\|^{2} \geq \nu_{1}, \quad \forall n \in \mathbb{N} .
$$

Setting $\nu_{2}:=\sup _{n \in \mathbb{N}}\left\|v_{n}\right\|^{2}<\infty$, it follows from (A.5-A.7) that

$$
c \geq d+\varepsilon_{n}-\varepsilon_{n} c d \frac{\nu_{2}}{\nu_{1}}, \quad \forall n \in \mathbb{N} .
$$

Since $\lim _{n \rightarrow \infty} \varepsilon_{n}=0$, this implies $c \geq d$, in contradiction to $d \in(c, b)$.

Corollary A.2. Suppose that $\phi \in \mathcal{S}[0, b)$ satisfies the assumptions (A3) and (A4) in Theorem 3.6 and let $R>0$. Then there exists an open neighbourhood $V \subset U$ of 0 , a number $c \in(a, b)$ and $\varepsilon>0$ such that $\phi \in \mathcal{S}[0, c] \cap \mathcal{S}_{V}[a, c]$ and the function $\psi: U \rightarrow U$ defined by

$$
\psi(v)= \begin{cases}\phi(v) & \text { if }\|v\| \leq R \\ c\left(1-\frac{R}{\|v\|}\right) v+\phi\left(\frac{R}{\|v\|} v\right) & \text { if } \quad\|v\|>R\end{cases}
$$

is in $\mathcal{S}[\varepsilon, b)$.

Proof: Since $\phi \in \mathcal{S}[0, b)$ and since $\phi$ satisfies assumption (A3), it is clear that there exist an open neighbourhood $V \subset U$ of 0 and a number $c \in(a, b)$ such that $\phi \in \mathcal{S}[0, c] \cap \mathcal{S}_{V}[a, c]$.

Let $d \in(c, b)$ such that $2 c-d>0$ and let $\delta \in(0,2 c-d)$. Setting

$$
b(v):=\phi\left(\frac{R}{\|v\|} v\right)-\frac{c R}{\|v\|} v
$$

we have for $v \in U$ with $\|v\|>R$,

$$
\left\|\psi(v)-\frac{d+\delta}{2} v\right\| \leq \frac{2 c-d-\delta}{2}\|v\|+\|b(v)\|=\frac{d-\delta}{2}\|v\|+(c-d)\|v\|+\|b(v)\| .
$$

Now $b: U \backslash\{0\} \rightarrow U$ is a bounded function, and so there exists $\tilde{R}>R$ such that

$$
\|b(v)\| \leq(d-c)\|v\|, \quad \forall v \in E_{\tilde{R}},
$$

where $E_{\tilde{R}}:=\{v \in U:\|v\| \geq \tilde{R}\}$. Combining this with (A.8) and applying (A.1) yields

$$
\psi \in \mathcal{S}_{E_{\tilde{R}}}[\delta, d] \subset \mathcal{S}_{E_{\tilde{R}}}[\delta, b) .
$$

To prove the claim, it remains to show that there exists $\varepsilon \in(0, \delta)$ such that

$$
\psi \in \mathcal{S}_{B_{\tilde{R}}}[\varepsilon, b),
$$

where $B_{\tilde{R}}:=\{v \in U:\|v\|<\tilde{R}\}$. This in turn will follow from Lemma A.1 if we can prove that $\psi \in \mathcal{S}[0, b)$ and $\psi$ satisfies assumptions (a) and (b) of Lemma A.1. To this end we proceed in three steps.

Step 1: We show that $\psi \in \mathcal{S}[0, b)$. Using the fact that $\phi \in \mathcal{S}[0, c]$, we obtain that for all $v \in U$ with $\|v\|>R$

$$
\left\|\psi(v)-\frac{c}{2} v\right\|=\left\|\frac{c}{2}\left(1-\frac{R}{\|v\|}\right) v+\phi\left(\frac{R}{\|v\|} v\right)-\frac{c R}{2\|v\|} v\right\| \leq \frac{c}{2}\left(1-\frac{R}{\|v\|}\right)\|v\|+\frac{c}{2} R=\frac{c}{2}\|v\| .
$$


Since $\psi(v)=\phi(v)$ for all $v \in U$ with $\|v\| \leq R$ and $\phi \in \mathcal{S}[0, c]$, it follows from (A.1) and (A.9) that $\psi \in \mathcal{S}[0, c] \subset$ $\mathcal{S}[0, b)$.

Step 2: Clearly, the set $V_{R}=\{v \in V:\|v\|<R\}$ is an open neighbourhood of 0 , and by construction

$$
\psi \in \mathcal{S}_{V_{R}}[a, b),
$$

showing that $\psi$ satisfies assumption (a) of Lemma A.1.

Step 3: To prove that $\psi$ satisfies assumption (b) of Lemma A.1, let $W \subset U$ be nonempty, bounded and closed with $0 \notin W$. Define $W_{R}:=\{w \in W:\|w\| \leq R\}$. Then $W_{R}$ is bounded and closed, and, if $W_{R} \neq \emptyset$, we obtain using assumption (A4) that

$$
\inf _{w \in W_{R}}\|\psi(w)\|=\inf _{w \in W_{R}}\|\phi(w)\|>0
$$

Furthermore, for all $w \in U$ with $\|w\| \geq R$

$$
\|\psi(w)\| \geq c\left(1-\frac{R}{2\|w\|}\right)\|w\|-\left\|\phi\left(\frac{R}{\|w\|} w\right)-\frac{c R}{2\|w\|} w\right\| \geq c\left(1-\frac{R}{2\|w\|}\right)\|w\|-\frac{c}{2} R=c(\|w\|-R),
$$

where in the second inequality we have used that $\phi \in \mathcal{S}[0, c]$. We see that for any $\eta>0$

$$
\inf _{\|w\| \geq R+\eta}\|\psi(w)\|>0
$$

Finally, for $\eta>0$, define

$$
B(R, \eta):=\{w \in U: R \leq\|w\| \leq R+\eta\} .
$$

By assumption (A4), $\inf _{\|v\|=R}\|\phi(v)\|>0$, and hence it follows that there exist $\gamma>0$ and $\eta^{*}>0$ such that for all $\eta \in(0, \eta *)$

$$
\inf _{w \in B(R, \eta)}\left\|\phi\left(\frac{R}{\|w\|} w\right)\right\| \geq \sup _{w \in B(R, \eta)}\left(c\left(1-\frac{R}{\|w\|}\right)\|w\|\right)+\gamma
$$

Therefore, since for all $w \in U$ with $\|w\| \geq R$

$$
\psi(w)=\phi\left(\frac{R}{\|w\|} w\right)+c\left(1-\frac{R}{\|w\|}\right) w
$$

we may conclude using (A.12) that

$$
\inf _{w \in B(R, \eta)}\|\psi(w)\| \geq \gamma>0, \quad \forall \eta \in\left(0, \eta^{*}\right) .
$$

Together with (A.10) and (A.11) this leads to

$$
\inf _{w \in W}\|\psi(w)\|>0
$$

showing that $\psi$ satisfies assumption (b) of Lemma A.1. 


\section{Part 2}

This part of the Appendix contains a proof of Lemma 4.4. We prove the following result of which Lemma 4.4 is an easy consequence.

Lemma A.3. Let $b>0$ and $f \in \mathcal{N}_{1}(b)$. If $f(0)=0$ and $f^{-}(0)>0$, then there exist constants $\varepsilon>0$ and $a \in(0, b)$ such that

i.e., $f \in \mathcal{S}_{(-\varepsilon, \varepsilon)}[a, b]$.

$$
a v^{2} \leq f(v) v \leq b v^{2}, \quad \forall v \in(-\varepsilon, \varepsilon),
$$

Proof: Since $f \in \mathcal{N}_{1}(b)$ and $f(0)=0$ it follows easily that

$$
f(v) v \leq b v^{2}, \quad \forall v \in \mathbb{R} .
$$

It remains to show that there exists $a \in(0, b)$ and $\varepsilon>0$ such that

$$
a v^{2} \leq f(v) v, \quad \forall v \in(-\varepsilon, \varepsilon) .
$$

Seeking a contradiction suppose that (A.13) is not true. Then there exist sequences $\left(v_{n}\right) \subset \mathbb{R} \backslash\{0\}$ and $\left(a_{n}\right) \subset(0, b)$ with $\lim _{n \rightarrow \infty} v_{n}=0$ and $\lim _{n \rightarrow \infty} a_{n}=0$ and such that

$$
a_{n} v_{n}^{2}>f\left(v_{n}\right) v_{n}
$$

Clearly, $\left(v_{n}\right)$ must contain a subsequence $\left(v_{n_{j}}\right)$ with either $v_{n_{j}}>0$ for all $j \in \mathbb{N}$ (Case 1) or $v_{n_{j}}<0$ for all $j \in \mathbb{N}$ (Case 2).

Case 1: Setting $\xi_{j}=h_{j}=v_{n_{j}}$, it follows that

$$
\frac{f\left(\xi_{j}-h_{j}\right)-f\left(\xi_{j}\right)}{h_{j}}=\frac{f(0)-f\left(\xi_{j}\right)}{\xi_{j}}=-\frac{f\left(\xi_{j}\right)}{\xi_{j}}>-a_{n_{j}} .
$$

This yields $f^{\circ}(0 ;-1) \geq 0$, and hence, $f^{-}(0) \leq 0$, contradicting the hypothesis that $f^{-}(0)>0$.

Case 2: Setting $\xi_{j}=0$ and $h_{j}=-v_{n_{j}}$, we have

$$
\frac{f\left(\xi_{j}-h_{j}\right)-f\left(\xi_{j}\right)}{h_{j}}=\frac{f\left(v_{n_{j}}\right)}{\left|v_{n_{j}}\right|}>a_{n_{j}} \frac{v_{n_{j}}}{\left|v_{n_{j}}\right|}=-a_{n_{j}} .
$$

Again, this yields $f^{\circ}(0 ;-1) \geq 0$, and hence, $f^{-}(0) \leq 0$, contradicting the hypothesis that $f^{-}(0)>0$.

We would like to thank Adam D. Mawby (Bath) for performing the simulations in Section 5.

\section{REFERENCES}

[1] M.A. Aizerman and F.R. Gantmacher, Absolute Stability of Regulator Systems. Holden-Day, San Francisco (1964).

[2] B.D.O. Anderson and S. Vongpanitlerd, Network Analysis and Synthesis: A Modern Systems Theory Approach. Prentice Hall, Englewood-Cliffs, NJ (1973).

[3] V. Barbu, Analysis and Control of Nonlinear Infinite-Dimensional Systems. Academic Press, Boston (1993).

[4] F. Bucci, Frequency-domain stability of nonlinear feedback systems with unbounded input operator. Preprint. Dipartimento de Matematica Applicata "G. Sansone", Università degli Studi di Firenze (1997) (to appear in Dynamics of Continuous, Discrete and Impulsive Systems).

[5] F.H. Clarke, Optimization and Nonsmooth Analysis. Wiley, New York (1983).

[6] F.H. Clarke, Yu.S. Ledyaev, R.J. Stern and P.R. Wolenski, Nonsmooth Analysis and Control Theory. Springer-Verlag, New York (1998).

[7] C. Corduneanu, Integral Equations and Stability of Feedback Systems. Academic Press, New York (1973). 
[8] C. Corduneanu, Almost Periodic Functions. Wiley, New York (1968).

[9] R.F. Curtain, H. Logemann, S. Townley and H. Zwart, Well-posedness, stabilizability and admissibility for Pritchard-Salamon systems. Math. Systems, Estimation and Control 7 (1997) 439-476.

[10] R.F. Curtain and G. Weiss, Well-posedness of triples of operators in the sense of linear systems theory, in Control and Estimation of Distributed Parameter System, edited by F. Kappel, K. Kunisch and W. Schappacher. Birkhäuser Verlag, Basel (1989) 41-59.

[11] G. Gripenberg, S.-O. Londen and O. Staffans, Volterra Integral and Functional Equations. Cambridge University Press, Cambridge (1990).

12] P.R. Halmos, Finite-Dimensional Vector Spaces. Springer-Verlag, New York (1987).

[13] H.K. Khalil, Nonlinear Systems, 2nd Edition. Prentice-Hall, Upper Saddle River, NJ (1996).

[14] S. Lefschetz, Stability of Nonlinear Control Systems. Academic Press, New York (1965).

[15] G.A. Leonov, D.V. Ponomarenko and V.B. Smirnova, Frequency-Domain Methods for Nonlinear Analysis. World Scientific, Singapore (1996).

[16] B.A.M. van Keulen, $H^{\infty}$-Control for Infinite-Dimensional Systems: A State-Space Approach. Birkhäuser Verlag, Boston (1993).

[17] H. Logemann, Circle criteria, small-gain conditions and internal stability for infinite-dimensional systems. Automatica $\mathbf{2 7}$ (1991) 677-690.

[18] H. Logemann and E.P. Ryan, Time-varying and adaptive integral control of infinite-dimensional regular linear systems with input nonlinearities. SIAM J. Control Optim. 38 (2000) 1120-1144.

[19] H. Logemann, E.P. Ryan and S. Townley, Integral control of linear systems with actuator nonlinearities: lower bounds for the maximal regulating gain. IEEE Trans. Auto. Control 44 (1999) 1315-1319.

[20] H. Logemann, E.P. Ryan and S. Townley, Integral control of infinite-dimensional linear systems subject to input saturation. SIAM J. Control Optim. 36 (1998) 1940-1961.

[21] H. Logemann and S. Townley, Low-gain control of uncertain regular linear systems. SIAM J. Control Optim. 35 (1997) 78-116.

[22] A. Pazy, Semigroups of Linear Operators and Applications to Partial Differential Equations. Springer-Verlag, New York (1983).

[23] W. Rudin, Functional Analysis. McGraw-Hill, New York (1973).

[24] D. Salamon, Realization theory in Hilbert space. Math. Systems Theory 21 (1989) 147-164.

[25] D. Salamon, Infinite-dimensional linear systems with unbounded control and observation: A functional analytic approach. Trans. Amer. Math. Soc. 300 (1987) 383-431.

[26] O.J. Staffans, Well-Posed Linear Systems, monograph in preparation (preprint available at http://www.abo.fi/ staffans/).

[27] O.J. Staffans, Quadratic optimal control of stable well-posed linear systems. Trans. Amer. Math. Soc. 349 (1997) $3679-3715$.

[28] M. Vidyasagar, Nonlinear Systems Analysis, 2nd Edition. Prentice Hall, Englewood Cliffs, NJ (1993).

[29] G. Weiss, Transfer functions of regular linear systems, Part I: Characterization of regularity. Trans. Amer. Math. Soc. 342 (1994) 827-854.

[30] G. Weiss, Admissibility of unbounded control operators. SIAM J. Control Optim. 27 (1989) 527-545.

[31] G. Weiss, Admissible observation operators for linear semigroups. Israel J. Math. 65 (1989) 17-43.

[32] G. Weiss, The representation of regular linear systems on Hilbert spaces, in Control and Estimation of Distributed Parameter System, edited by F. Kappel, K. Kunisch and W. Schappacher. Birkhäuser Verlag, Basel (1989) 401-416.

[33] D. Wexler, On frequency domain stability for evolution equations in Hilbert spaces via the algebraic Riccati equation. SIAM J. Math. Analysis 11 (1980) 969-983.

[34] D. Wexler, Frequency domain stability for a class of equations arising in reactor dynamics. SIAM J. Math. Analysis 10 (1979) 118-138. 